\title{
ఠThe Role of Random Vorticity Stretching in Tropical Depression Genesis $\mathscr{O}$
}

\author{
HaO FU'a AND MORGAN O'NeILL ${ }^{\mathrm{a}}$

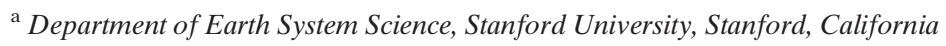

(Manuscript received 21 March 2021, in final form 5 August 2021)

\begin{abstract}
Tropical deep convection plays a key role in the tropical depression stage of tropical cyclogenesis by aggregating vorticity, but no existing theory can depict such a stochastic vorticity aggregation process. A vorticity probability distribution function (PDF) is proposed as a tool to predict the horizontal structure and wind speed of the tropical depression. The reason lies in the tendency for a vortex to adjust to an axisymmetric and monotonic vorticity structure. Assuming deep convection as independent and uniformly distributed vortex tube stretching events in the low-midtroposphere, repetitive vortex tube stretching will make the air column area shrink many times and significantly increase vorticity. A theory of the vorticity PDF is established by modeling the random stretching process as a Markov chain. The PDF turns out to be a weighted Poisson distribution, in good agreement with a randomly forced divergent barotropic model (weak temperature gradient model), and in rough agreement with a cloud-permitting simulation. The result shows that a stronger and sparser deep convective mode tends to produce more high-vorticity air columns, which leads to a more compact major vortex with a higher maximum wind. Based on the vorticity PDF theory, a parameterization of the eddy acceleration effect on the tangential flow is proposed.
\end{abstract}

KEY WORDS: Deep convection; Stochastic models; Tropical cyclones

\section{Introduction}

The mechanism of tropical cyclogenesis remains a challenging scientific problem. The formation of a tropical cyclone might be loosely split into three stages: (i) the formation of a weak cyclonic region ("embryo region") where convection prefers to occur, (ii) the spinup of a cyclonic circulation in the embryo, and (iii) its intensification into a vortex with a selfsustaining eyewall updraft (Montgomery et al. 2006).

The embryo region usually takes the form of one or multiple mesoscale convective systems (MCS). It can be generated in an easterly wave trough (Gray 1998) and especially its intersection with the critical layer (Dunkerton et al. 2009), a large-scale confluence zone, a monsoon shear line (Ritchie and Holland 1999), a pair of mixed Rossby-gravity waves' cyclonic gyres (Ferreira et al. 1996), or perhaps spontaneously via the self-aggregation of deep convection (Bretherton et al. 2005; Wing et al. 2016; Holloway et al. 2017). The MCS provides a moist region which protects the convection inside from laterally entraining dry air. Its weak midlevel cyclonic circulation also protects convection from the environmental strain and provides some additional basic-state background vorticity for convection to stretch (Dunkerton et al. 2009).

At the second stage, deep convection produces vertical vorticity anomaly in the lower and middle troposphere due to

\footnotetext{
OSupplemental information related to this paper is available at the Journals Online website: https://doi.org/10.1175/JAS-D-210087.s1.

๑Denotes content that is immediately available upon publication as open access.
}

Corresponding author: Hao Fu, haofu736@gmail.com vortex tube stretching and tilting, which is confirmed in both numerical simulation and observation (Montgomery et al. 2006; Bell and Montgomery 2019). The convection usually dies in around an hour, but the vorticity remnant lasts far longer (Wissmeier and Smith 2011), and can be involved in another stretching event to produce higher vorticity (Kilroy et al. 2017). The vorticity patches are further pushed inward by the midlevel converging overturning circulation (Kilroy et al. 2017), which is driven by Ekman pumping, moisture, and longwave radiation feedbacks (Smith 2000; Davis 2015). Montgomery et al. (2006) intuitively described it as 2D turbulence punctuated by $3 \mathrm{D}$ convective events. In about 2 days, the subsequent vortex merger spins up a tropical depression which is a quasi-circular vortex with a sustained maximum surface wind of up to $17 \mathrm{~m} \mathrm{~s}^{-1}$, but generally free of the eye (Charney and Eliassen 1964; Montgomery et al. 2006; American Meteorological Society 2012).

At the third stage, the deepened central pressure (corresponding to a high-inertial-stability core), Ekman pumping and the strong surface heat flux near the radius of maximum wind lead to a ringlike eyewall updraft, marking the maturity of the hurricane (Rotunno and Emanuel 1987; Emanuel 1997; Gray 1998). The midlayer contraction slows down due to the establishment of gradient wind balance, and the inflow enters mostly through the frictional boundary layer (Montgomery and Smith 2014).

We focus on the second stage, which is the spinup phase of the vortex, or in other words, the formation of tropical depression from an existing MCS. As for theoretical modeling, Charney and Eliassen (1964) studied the role of Ekman pumping in lifting parcels and releasing the stored conditional instability. Based on Emanuel (1986), there is a series of axisymmetric balanced models with a prognostic boundary layer moist entropy and angular momentum, which controls the free troposphere via convective quasi-equilibrium assumption 
(Rotunno and Emanuel 1987; Emanuel 1989, 2012). They showed that the wind-induced surface sensible and latent heat fluxes moisten the atmosphere, weaken downdrafts, and enhance updrafts as the wind grows to finite amplitude. Raymond et al. (2007) used gross moist stability (Neelin and Held 1987) to link wind-induced surface heat flux to convergence and explored its competition with the wind-induced frictional spindown rate. All three models solve the axisymmetric mode. They do not include an important asymmetric effect: the aggregation of convectively generated eddies, which has been recognized as "vortical hot tower route" (Hendricks et al. 2004; Montgomery et al. 2006). The spinup is due to the cooperation between the upscale growth of the convectioninduced vorticity, as well as the inward advection by the convection-induced inflow above the boundary layer (Kilroy et al. 2017). In an axisymmetry/asymmetry view, the acceleration of the azimuthal flow by eddies is represented by the radial eddy transport flux of vorticity (Montgomery and Kallenbach 1997; Nolan and Farrell 1999; Hendricks et al. 2004), but it has not been successfully parameterized yet. In a stochastic view, it has been shown with simulation that the vorticity stretching occurs almost randomly and can produce high-vorticity patch by accumulation (Kilroy et al. 2017). The stochasticity also limits the predictability (Van Sang et al. 2008). However, the random stretching remains a descriptive concept.

In this paper, we present a novel perspective for understanding the vortex spinup by deep convection: considering the widening of vertical vorticity probability distribution function (PDF) through the repetitive random stretching and convergent transport by the short-lived convective clouds, and the subsequent rearrangement of the vorticity field into an axisymmetric and radially monotonic compact vortex by the eddies. We will show that the PDF evolution roughly obeys a Markov chain which yields an analytical solution. The axisymmetric state may not be completely reached by the end of the second stage, but it sets an upper bound of maximum wind that can be reached in the axisymmetrization process. The complicated eddy dynamics only determines the axisymmetrization time scale and a modification of the PDF by turbulent mixing, which is the second step to consider. We build a onelayer shallow water model to demonstrate it, with random local mass sink seeded into a circular region to mimic an existing MCS. This scheme, which has been used in a domainhomogeneous seeding mode, was first introduced by Vallis et al. (1997) and has been applied to study the formation of the Great Red Spot (Showman 2007), the giant planets' polar vortex (O'Neill et al. 2015, 2016) and jet (Thomson and McIntyre 2016). To the authors' knowledge, the scheme has not been applied to tropical cyclogenesis where convection concentrates in a portion of the domain, and its elegant statistical property as a Markov chain remains untouched.

The paper is organized in the following way. Section 2 introduces the simplified tropical depression genesis setup within a shallow water equation (SWE) and its further simplification to a weak temperature gradient model where the Markov chain is more strictly valid. Section 3 describes the numerical solver. Section 4 describes the flow evolution of the reference test. Section 5 presents a theory of the vorticity PDF. Section 6 validates the PDF model with sensitivity tests and discusses its link to vortex intensity. Section 7 further validates the theory with a cloud-permitting simulation of tropical cyclogenesis. Section 8 concludes the paper. The derivation of the continuous PDF equation and a list of mathematical symbols are presented in the online supplemental material.

\section{An idealized tropical depression genesis problem}

\section{a. The macroscopic setup}

First, we present a more detailed review of the environment of tropical depression formation, which guides the design of a shallow water setup. An easterly wave trough that provides a cyclonic background relative vorticity is of $\sim 700-\mathrm{km}$ scale (Gray 1998). It can embed multiple $\sim 250-\mathrm{km}$-scale MCSs, which are a mixture of aggregated stratiform and convective cloud (Gray 1998). An MCS usually possesses a midlevel cyclonic circulation which is strongest at about 5-km height (Fritsch et al. 1994; Houze et al. 2009). An MCS usually lacks near-surface vertical vorticity, due to the divergence caused by the low-level $(<2 \mathrm{~km})$ evaporative cooling of precipitation falling from the stratiform region (Fritsch et al. 1994). If there is growth of low-level vorticity, air-sea interaction feedback will be excited and the system will be on the track of tropical cyclogenesis.

The formation mechanism of low-level vorticity is still in debate. One explanation is the "top-down" development of the midlevel vortex via vortex interaction (Ritchie and Holland 1997) or transport by evaporation-driven downdraft (Bister and Emanuel 1997). The other is the "bottom-up" mechanism. It emphasizes the production of low-level vorticity by the deep convective stretching of the MCS's vertical absolute vorticity, which is small but nonzero at low level (Montgomery et al. 2006). The deep convection is promoted by the moistening and cooling of the lower and middle levels by the MCS' stratiform precipitation (Bell and Montgomery 2019).

In this paper, we focus on the spinup of the low-midlevel ( 1-6-km height) by deep convective vorticity stretching within an MCS, which is the basis of the "bottom-up" view but largely remains descriptive. According to Kilroy et al. (2017), this is the level where most of the free-tropospheric convergence occurs. The influence of the existing midlevel relative vorticity, as well as the upward transport of boundary layer vorticity during Ekman pumping, are neglected. The lowmidlevel is modeled with a one-layer barotropic model. We seed the updrafts only in a circular "vigorous convection region" with a fixed radius of $R(\sim 100 \mathrm{~km})$, to mimic a single MCS in a doubly periodic domain whose width is $L \sim 800 \mathrm{~km}$. A schematic diagram of this setup is presented in Fig. 1. During the spinup of a realistic tropical depression, the midlayer inflow that is characterized by the MCS region mean divergence $\delta_{0}$ (negative) first grows due to higher tropospheric moisture and surface flux, and then decreases due to the buildup of inertial stability (Kilroy et al. 2017). The $\delta_{0}$ depends on the complicated interaction between convection and the vortex. For simplicity, we will use a fixed $\delta_{0}$ in the theoretical model of the vorticity PDF and all the one-layer model 


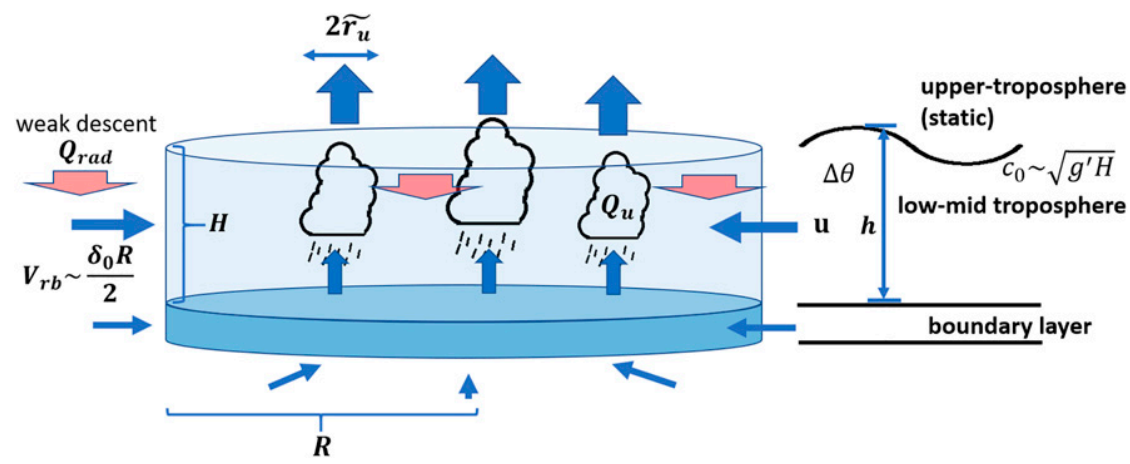

FIG. 1. A schematic diagram of the mesoscale convective system (MCS) and the shallow water setup, which depicts the low-middle troposphere. Deep convection only occurs in the MCS. The air entrained by convection to the upper troposphere returns to the low-middle troposphere via a uniform radiative cooling.

simulations. However, it will be shown that the PDF theory does not require this constraint: the time dependence of $\delta_{0}$ can be absorbed into a nondimensional time coordinate that is rescaled with $\delta_{0}$. This novel rescaling decouples the convective feedback from vortex dynamics to some extent.

\section{b. The shallow water analogy for a stratified atmosphere}

We follow Hendricks et al. (2014) to view the continuously stratified atmosphere as a one-layer isentropic model (barotropic model). The active layer represents the low-midtroposphere (roughly 1-6-km height), with a thickness of $H=5 \mathrm{~km}$ and a vertical temperature range of $\Delta \theta=22.04 \mathrm{~K}$. It is capped by a static upper layer which denotes the upper troposphere. As the atmospheric lapse rate in the tropics is close to moist adiabatic, we set the reduced gravity as $g^{\prime}=g \Delta \theta / \theta_{00}=0.72 \mathrm{~m} \mathrm{~s}^{-2}$, with $\theta_{00}=300 \mathrm{~K}$ denoting a reference potential temperature. The internal gravity wave speed is $c_{0}=\sqrt{g^{\prime} H}=60 \mathrm{~m} \mathrm{~s}^{-1}$.

Ooyama (1969) presented a three-layer view of deep convection, which is recently updated by Schecter and Dunkerton (2009). Suppose the boundary layer detrains a thermal bubble. It releases latent heat, entrains some midtropospheric air, and will rise to the upper troposphere if it is buoyant enough. For the even simpler one-layer setup, we let the low-midlevel-tropospheric thickness sink due to the convective heating be $Q_{u}$ (negative, unit: $\mathrm{m} \mathrm{s}^{-1}$ ). The evaporative cooling induced by stratiform cloud does project onto the lowmidtroposphere, but is neglected here. Parcels are assumed to slowly return from the upper to the low-middle layers through radiative cooling, which corresponds to a thickness source $Q_{\text {rad }}$ (positive, unit: $\mathrm{m} \mathrm{s}^{-1}$ ). The continuity and momentum equations are

$$
\begin{gathered}
\frac{\partial h}{\partial t}+\nabla \cdot(\mathbf{u} h)=Q_{u}+Q_{\mathrm{rad}}+\nu \nabla^{2} h, \\
\frac{\partial \mathbf{u}}{\partial t}+\mathbf{u} \cdot \nabla \mathbf{u}+f_{0} \mathbf{k} \times \mathbf{u}=-g^{\prime} \nabla h-\frac{\mathbf{u}}{\tau_{d}}+\nu \nabla^{2} \mathbf{u} .
\end{gathered}
$$

Here $\mathbf{u}$ is the horizontal velocity vector, $h$ is layer thickness, $f_{0}$ is Coriolis parameter, $\nabla \equiv \mathbf{i} \partial / \partial x+\mathbf{j} \partial / \partial y$ is the horizontal gradient operator. For simplicity, we treat the Ekman spindown, as well as the vertical momentum transfer by both updraft (cumulus drag) and radiative cooling, as a bulk linear Rayleigh drag whose time scale is $\tau_{d}$. The $\nu$ is a constant diffusivity to suppress numerical instability. To be self-consistent with the omission of cumulus drag, an updraft is assumed to move with the low-midlevel wind. This is different from the shallow water models of Showman (2007) and O'Neill et al. (2016), who fix the updraft position in a convective event.

In the real atmosphere, as the vortex intensifies, the lowlevel horizontal vorticity could be tilted by the updraft to form vertical vorticity dipoles (Vallis et al. 1997). These, together with the vorticity monopoles produced by stretching, are both important for hurricane formation (Montgomery et al. 2006; Kilroy et al. 2014). What is more, the Ekman pumping causes divergence in the middle troposphere, which can counteract part of the convection-induced inflow (Smith 2000). We will leave the careful consideration of these processes for future work.

\section{c. The application of weak temperature gradient approximation}

We propose that SWE can be further simplified to a weak temperature gradient (WTG) equation for understanding the vortex spinup at the early stage of tropical cyclogenesis. It is vital for establishing the vorticity PDF model in section 5.

The Coriolis force and the fluid inertia tend to invoke some pressure gradient to balance them. At the low latitude where the Coriolis parameter is small, the buoyancy anomaly associated with the gravest mode (convective heating mode) cannot accumulate at the large scale. This leads to WTG approximation, where geostrophic adjustment is instantaneous and the continuity equation in SWE simplifies to the balance between mass sink and divergence (Sobel et al. 2001):

$$
H \delta=Q_{u}+Q_{\mathrm{rad}}
$$

Here $\delta=\nabla \cdot \mathbf{u}$ is horizontal divergence which is externally prescribed with convective and radiative parameterization. Dynamically, the potential vorticity degenerates to absolute vorticity $\omega_{a}=\omega+f_{0}$ where $\omega$ is relative vorticity (Sobel et al. 2001).

Enagonio and Montgomery (2001) have shown that there is little difference between a shallow water model and the 
nondivergent barotropic model $(\delta=0)$ for the free-evolving $2 \mathrm{D}$ vortex dynamics of an early stage hurricane vortex, primarily due to (i) the small system length scale compared to Rossby deformation radius of the gravest mode, (ii) the small Froude number Fr (definition shown below), and (iii) the fast geostrophic adjustment compared to the axisymmetrization time scale (measured with strain rate). As the convergent flow is much weaker than the rotational flow, the criterion for WTG should be identical. The first two criteria which are more basic are stated in (4) and (5), respectively:

$$
\begin{gathered}
\mathrm{Bu}=\frac{R^{2}}{L_{R}^{2}} \ll 1, \quad \text { with } \quad L_{R}=\frac{\sqrt{g^{\prime} H}}{f_{0}}, \\
\mathrm{Fr}=\frac{V_{\theta}}{\sqrt{g^{\prime} H}} \sim\left(-\frac{h^{\prime}}{H}\right)^{1 / 2} \ll 1,
\end{gathered}
$$

where $L_{R}$ is Rossby deformation radius, $h^{\prime}$ is the deviation of layer thickness from its basic state $H, F r$ is defined as the ratio of azimuthal wind scale $V_{\theta}$ to gravity wave speed $c_{0}$. Using $R=100 \mathrm{~km}$ and a low latitude Coriolis parameter $f_{0}$ $\sim 5 \times 10^{-5} \mathrm{~s}^{-1}$, we get $L_{R} \sim 1200 \mathrm{~km}$ and Burger number $\mathrm{Bu}=0.007 \ll 1$. The relation between Fr and $-h^{\prime} / H$ in $(5)$ is derived with cyclostrophic wind balance assumption, which is applicable to a strong tropical depression where Coriolis force is generally weaker than centrifugal acceleration. It is worth noting that gravity wave will be important to hurricane when it is coupled to convection (Lahaye and Zeitlin 2016).

The full WTG governing equation is introduced here. In the updraft region, the mass loss is balanced by convergence immediately; radiative cooling drives a slow divergent flow everywhere. The vertical vorticity equation is

$$
\frac{\partial \omega}{\partial t}+\mathbf{u} \cdot \nabla \omega=-\delta\left(\omega+f_{0}\right)-\frac{\omega}{\tau_{d}}+\nu \nabla^{2} \omega
$$

The total velocity is a superposition of rotational wind and divergent wind:

$$
\mathbf{u}=\mathbf{k} \times \nabla \psi+\nabla \phi, \quad \text { with } \quad \nabla^{2} \psi=\omega, \nabla^{2} \phi=\delta .
$$

Here $\psi$ is a streamfunction and $\phi$ is a velocity potential. They are linked to $\omega$ and $\delta$ through the two Poisson equations. The doubly periodic boundary condition eliminates the harmonic component. Equations (3), (6), and (7) are the so-called weak temperature gradient approximation equation (WTGE). It is essentially a 2D barotropic vorticity equation punctuated by diabatic vorticity stretching, which involves both vorticity magnitude change and a convergent flow.

\section{d. The convective representation}

\section{1) FORMULATION}

We employ the random convection scheme of Showman (2007) in simulating convection on Jupiter to study the terrestrial tropical atmosphere. The convective mass sink rate (or equivalently the midlayer updraft velocity increment with a minus sign) $Q_{u}$ is represented as a sequence of Gaussian shape mass sinks $Q_{n}$ (unit: $\mathrm{m} \mathrm{s}^{-1}$ ) that are isolated in time and space:

$$
\begin{gathered}
Q_{u}(\mathbf{x}, t)=\sum_{n=1}^{+\infty} Q_{n}(\mathbf{x}, t), \quad \text { with } \\
Q_{n}(\mathbf{x}, t)=\widetilde{Q_{m}} e^{-\left|t-t_{n}\right|^{2} /{\widetilde{\tau_{u}}}^{2}-\left|\mathbf{x}-\mathbf{x}_{n}\right|^{2} /{\widetilde{r_{u}}}^{2}} .
\end{gathered}
$$

For every $\Delta t$ time, a new updraft is seeded to a random position in a circular area within a radius $R$ which represents an MCS. The $\widetilde{Q_{m}}$ is the peak mass sink rate, $\widetilde{\tau_{u}}$ is a fixed updraft duration time scale which is controlled by the onset time of downdraft in the real atmosphere (Emanuel 1994), and $\widetilde{r_{u}}$ is a fixed updraft size parameter. The seeding time is a deterministic sequence: $t_{n}=n \Delta t$. The updraft center vector $\mathbf{x}_{n}$ moves with the local wind. It is calculated with the initial seeding position $\mathbf{x}_{0 n}$, which is a random vector sequence with uniform distribution that satisfies $\left|\mathbf{x}_{0 n}\right|<R$ (with the MCS center as the coordinate origin), as well as the local velocity: $\mathbf{x}_{n}=\mathbf{x}_{0 n}+\int_{t_{n}}^{t} \mathbf{u}\left[\mathbf{x}\left(t^{\prime \prime}\right), t^{\prime \prime}\right] d t^{\prime \prime}$.

The radiative cooling $Q_{\text {rad }}$ (positive) is set as a spatially uniform but temporally fluctuating value that instantaneously balances $Q_{u}$ to keep the domain average diabatic heating at zero:

$$
Q_{\mathrm{rad}}=-\frac{1}{L^{2}} \iint Q_{u} d \mathbf{x} \approx-\delta_{0} H \frac{\pi R^{2}}{L^{2}} .
$$

The fluctuation, which is manifested as the “ $\approx$ " symbol in (9), converges to a constant value if there are enough clouds in the domain. For WTGE, the total divergence induced by convective and radiative process obeys (3).

The diabatic heating that involves random factors leads to a set of stochastic differential equations. This convective scheme offers adjustable cloud parameters. For smaller updraft strength $-\widetilde{Q_{m}}$ and seeding interval $\Delta t$, convection is more fine-grained and the limit is a purely axisymmetric and deterministic problem controlled by the convergence of angular momentum rings.

Physically, the homogeneous updraft seeding within the MCS mostly originates from the seemingly random boundary layer dry convection or cold pool collision (Würsch and Craig 2014; Haerter 2019; Torri and Kuang 2019). However, it is increasingly unsuitable as the major vortex develops, mainly in three aspects:

- The convective position should be allowed to follow the contracting high-vorticity region which pumps more air out of the boundary layer. The coupling of convection to local vorticity is also needed to properly represent the convectively coupled vortex Rossby wave and its interaction with mean flow (Wang 2002a,b).

- The convective strength near the vortex center should be allowed to be suppressed by the upper tropospheric warm core which is mainly induced by centrifugal acceleration (Schecter and Dunkerton 2009). This effect will be important when the layer thickness gradient is large (Fr is large). 
- The midlayer entrainment might decrease as the environment spins faster (higher inertial stability). This is a robust behavior of dry rotating plumes but is still unclear for moist convection (Julien et al. 1999; Peters et al. 2020).

\section{2) STATISTICAL PROPERTY}

For convenience, we idealize the Gaussian updraft as a "mass-equivalent top-hat" with a mass sink of $\widetilde{Q_{m}}$, radius $\widetilde{r_{u}}$, and duration time $\widetilde{T_{u}}=\pi^{1 / 2} \widetilde{\tau}_{u}$. We will introduce the "vorticity equivalent" top-hat in section $4 \mathrm{a}$, which is an approximation to a sufficiently diffused "mass-equivalent tophat." To distinguish between the two objects, the tilde $(\sim)$ symbol is used to denote parameters related to "mass-equivalent top-hat." These parameters can be combined into two divergence scales, which are the divergence at an updraft $\delta_{u}$ $(<0)$, and the MCS mean divergence $\delta_{0}(<0)$ which is much smaller in magnitude:

$$
\delta_{u}=\frac{\widetilde{Q_{m}}}{H}, \quad \delta_{0}=\frac{\widetilde{Q_{m}}}{H} \frac{\widetilde{r_{u}}}{R^{2}} \frac{\widetilde{T_{u}}}{\Delta t} .
$$

The statistically averaged time interval between the onset of two convective events at a fixed location (revisit time) is $\tau_{\text {rev }}=\Delta t R^{2} /{\widetilde{r_{u}}}^{2}$. It leads to two important nondimensional parameters:

- Nondimensionalizing $\tau_{\text {rev }}$ with $\delta_{0}$ yields the convective intermittency parameter, which turns out to be the accumulated convergence of an updraft:

$$
-\frac{\widetilde{\Delta h}}{H}=-\delta_{0} \tau_{\mathrm{rev}}=-\frac{\widetilde{Q_{m}} \widetilde{T_{u}}}{H}=\frac{-\delta_{0} \Delta t R^{2}}{\widetilde{r_{u}}{ }^{2}} .
$$

Here $\widetilde{\Delta h}=\widetilde{Q_{m}} \widetilde{T_{u}}$ is the layer thickness change in an updraft. A higher $-\widetilde{\Delta h} / H$ means convection is in a more coarsegrained mode. The probability for any particular location to be under convection, or equivalently, the updraft fractional area is $\sigma_{u}=\widetilde{T}_{u} / \tau_{\mathrm{rev}}=\delta_{0} / \delta_{u}$. The $\sigma_{u}$ is also the probability for the center of a new updraft to fall on the convective region, so it measures the potential of updraft interaction.

\section{The numerical method and the reference test}

The SWE, which is defined in (1) and (2), as well as the WTGE, which is defined in (3), (6), and (7), are solved on a doubly periodic square plane. The numerical solver is a MATLAB code developed by the first author. It uses standard spectral method, with zero padding technique to eliminate the aliasing error in product terms. The SWE solver uses the semi-implicit second-order leapfrog time stepping to stabilize the fast gravity wave, and the WTGE solver (without concerning gravity wave) uses the third-order Runge-Kutta time stepping which has higher temporal accuracy (Durran 2010).

In appendix A, we show that the SWE and WTGE can be fully nondimensionalized. The WTGE is controlled by six nondimensional parameters: $-\delta_{0} / f_{0}$ measures the relative importance of the convergent to rotational dynamics, $-\widetilde{\Delta h} / H$ measures convective intermittency, $-\delta_{0} \widetilde{T}_{u}$ measures convective time scale, $\widetilde{r_{u}} / R$ measures the updraft size relative to the system size, $-\delta_{0} \tau_{d}$ measures the strength of drag, and $\mathrm{Re}=-\delta_{0} R^{2} / \nu$ measures the importance of horizontal viscosity. For the reference test, we set $-\delta_{0} / f_{0}=0.228$, $-\widetilde{\Delta h} / H=8 / 5,-\delta_{0} \widetilde{T_{u}}=0.04, \widetilde{r_{u}} / R=0.08,-\delta_{0} \tau_{d}=\infty$ (no damping), and $\operatorname{Re}=711.13$. For the SWE, there is an additional $\mathrm{Bu}=R^{2} / L_{R}^{2}=0.007$.

A dimensional interpretation of the reference test is shown below. It corresponds to a domain width of $L=800 \mathrm{~km}$, a $20^{\circ} \mathrm{N}$ Coriolis parameter $f_{0}=4.99 \times 10^{-5} \mathrm{~s}^{-1}$, a system-averaged convergence $\delta_{0}=-1.138 \times 10^{-5} \mathrm{~s}^{-1}\left(-\delta_{0}^{-1}=1.02\right.$ days $)$, a basicstate layer thickness $H=5 \mathrm{~km}$, an updraft total thickness sink $\widetilde{\Delta h}=-8 \mathrm{~km}$, an updraft duration time $\widetilde{\tau_{u}}=2000 \mathrm{~s}$ $\left(\widetilde{T_{u}}=3544.9 \mathrm{~s}\right)$ and an updraft radius $\widetilde{r_{u}}=8 \mathrm{~km}$ based on the estimation of Hendricks et al. (2004) and Montgomery et al. (2006), an MCS radius $R=100 \mathrm{~km}$ (Houze et al. 2009), and a horizontal viscosity $\nu=160 \mathrm{~m}^{2} \mathrm{~s}^{-1}$ which is approximately the lowest $\nu$ to guarantee numerical stability at current resolution $(576 \times 576$ grid points in physical space, or a grid spacing of $1.39 \mathrm{~km}$ ). Other parameters can be calculated from the settings above: $\widehat{Q_{m}}=-2.26 \mathrm{~m} \mathrm{~s}^{-1}, \Delta t=900 \mathrm{~s}, \sigma_{u}=0.025$. In numerical implementation, the integration time is 4 days, and the time step is $4.96 \mathrm{~s}$. The Courant number estimated with a $30 \mathrm{~m} \mathrm{~s}^{-1}$ wind is 0.11 . As the model is inherently stochastic, each test includes an ensemble of 20 runs.

We remark that $-\delta_{0} / f_{0}$ is regarded as a free parameter in this model. Physically, it depends on the complicated feedback between convection and the vortex circulation, and should at least depend on $R$ and $f_{0}$. The current choice of $\delta_{0}$ enables the system to exceed the $17 \mathrm{~m} \mathrm{~s}^{-1}$ tropical depression bound and reach the tropical storm stage in three days, which is not far from the 1-2 days growth time scale in the real atmosphere (Montgomery et al. 2006).

\section{The flow evolution}

We first consider the vorticity production process in a single updraft, and then move to the tropical depression genesis problem which involves multiple updrafts.

\section{a. Single convection}

A convective updraft induces a convergent flow that reduces the air column area, and increases the vorticity by stretching. In an updraft event with an accumulated convergence of $-\widetilde{\Delta h} / H$, the area of air involved in a stretching process shrinks from $\pi{\widetilde{r_{u}}}^{2}(1-\widetilde{\Delta} h / H)$ to $\pi{\widetilde{r_{u}}}^{2}$. The conservation of absolute circulation shows that the average absolute vorticity within the influenced area changes from an initial value $\omega_{a, 0}$ to $\omega_{a, 0}(1-\widetilde{\Delta h} / H)$. Though the vorticity does not diminish after the updraft event, the size reduction induces a stronger horizontal diffusion that smooths the vorticity anomaly. In the vorticity PDF model, we are interested in the vorticity strength by the time it is hit by the next updraft, so a diffusion-correction should be implemented. 
In appendix B, we use scale analysis to show that a significant smoothing does occur. We introduce a "vorticity-equivalent top-hat," which is a flatter version of the "massequivalent top-hat" that describes the shape of the vorticity patch seen by the next convection. The vorticity top-hat radius is set as $r_{u}=\alpha_{r} \widetilde{r_{u}}$, where $\alpha_{r}$ is a free parameter. The total absolute circulation within $r_{u}$ is still conserved. In section 6a, we show that a fixed $\alpha_{r}=\sqrt{2}$ works well in the vorticity PDF model. We preliminarily explain the insensitivity as an automatic damping mechanism: diffusion only exerts strong damping when the scale shrinks to a small enough value. For example, a low Re case has strong diffusion, but the damping becomes weak when the previous diffusion has sufficiently damped the sharp structure.

Letting the "vorticity-equivalent accumulated convergence" $-\Delta h$ be the average accumulated convergence within a radius of $r_{u}$, we have $\Delta h=\widetilde{\Delta h} / \alpha_{r}^{2}=\widetilde{\Delta h} / 2$. There is $r_{u}=8 \sqrt{2} \mathrm{~km}$ and $\Delta h=-4 \mathrm{~km}$ for the reference test. Based on (11), the new convective intermittency parameter to be frequently used is

$$
\frac{\Delta h}{H}=\delta_{0} \Delta t \frac{R^{2}}{r_{u}^{2}} .
$$

The smoothed absolute vorticity within $r_{u}$ is denoted as $\left[\omega_{a}\right]^{r_{u}}$, which is calculated with the conservation of absolute circulation

$$
\left[\omega_{a}\right]^{r_{u}}=\omega_{a, 0}\left(1-\frac{\Delta h}{H}\right) .
$$

\section{b. Multiple convection}

\section{1) THE EVOLUTION OF THE AVERAGE VORTICITY}

As a macroscopic constraint, the mean low-midlevel relative vorticity of the MCS (defined as $\omega^{+}$) is directly related to the MCS mean divergence $\delta_{0}$ and damping effects, based on Gauss theorem (Raymond et al. 2007; Montgomery and Smith 2017). It does not depend on how noisy the deep convection is. As the vorticity gradient at the MCS boundary is small, horizontal diffusion is unimportant. Raymond et al. (2007) have used this noise-independent property to establish their model of system-averaged vorticity which includes an interactive $\delta_{0}$ and a quadratic drag that both increase with the mean vorticity.

When there is a fixed $\delta_{0}$ and no drag, a large enough domain $(L \gg R)$ yields

$$
\omega^{+} \approx-f_{0} \delta_{0} t
$$

This is demonstrated in Fig. 2a. The large domain dilutes the compensating divergence, so the vorticity squashing and therefore the negative relative vorticity outside of the MCS is small. Thus, the relative vorticity transported into the MCS is close to zero-a key assumption to be used in our vorticity PDF model. In appendix C, we include Rayleigh drag into this problem, and consider a finite domain correction to (14). The drag directly damps $\omega^{+}$, and the negative relative vorticity transported into the MCS indirectly damps $\omega^{+}$.
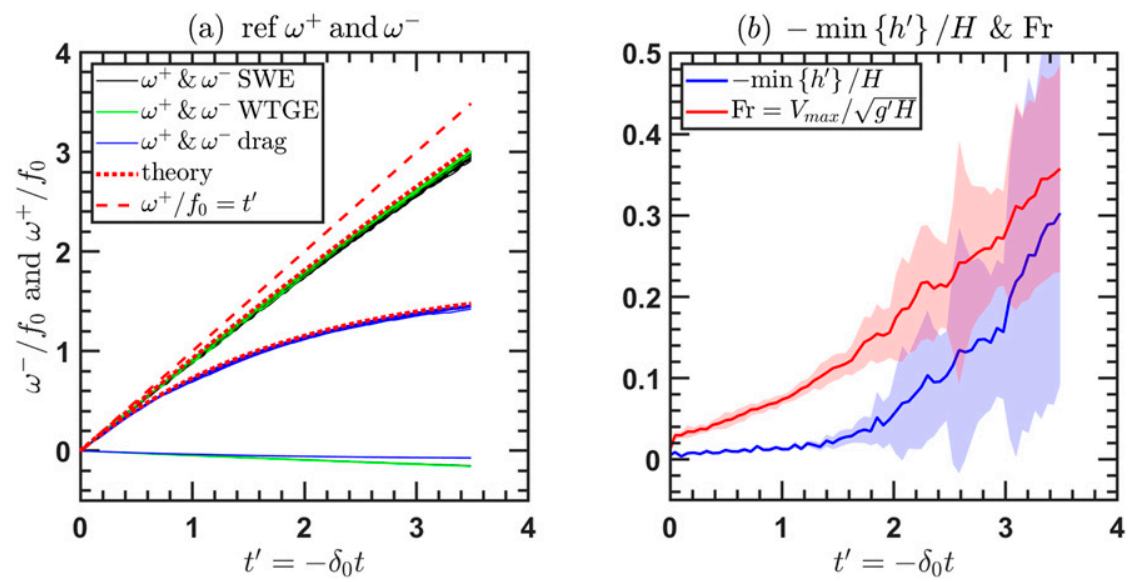

FIG. 2. (a) The time evolution of the mean vorticity in the MCS $\left(\omega^{+}\right)$and out of the MCS $\left(\omega^{-}\right)$for an ensemble of 20 runs of the SWE reference test (Ref-SWE, 20 solid black lines), the WTGE reference test (Ref-WTGE, 20 solid green lines), the $-\delta_{0} / \tau_{d}=2$ drag sensitivity test (20 solid blue lines), the finite-domain theory of $\omega^{+}$in (C5) for both the reference and drag test (2 dotted red lines), and the approximate solution $\omega^{+} / f_{0}=-\delta_{0} t$ (dashed red line). Note that all of the $\omega^{+} / f_{0}$ data are positive, and that of $\omega^{-} / f_{0}$ are negative. The time coordinate $t^{\prime}$ is the time nondimensionalized with the MCS mean divergence: $t^{\prime}=-\delta_{0} t$. (b) The blue line shows the time evolution of the domain minimum thickness disturbance normalized by the basic-state thickness: $-\min \left\{h^{\prime} / H\right\}$ for an ensemble of 20 runs of the SWE reference test (Ref-SWE). The red line shows the ensemble-averaged $\mathrm{Fr}=V_{\max } / \sqrt{g^{\prime} H}$, where $V_{\max }$ is the maximum total wind. The shadow denotes the \pm 1 standard deviation range. For both (a) and (b), only the data of $0 \leq t^{\prime} \leq 3.56$ are plotted, because one SWE run blows up after that, due to the computational instability induced by the sharp vorticity gradient. 
To understand the maximum wind, we still need to know how $\omega^{+}$is distributed within the MCS, which involves the dynamics of eddies.

\section{2) THE EVOLUTION OF EDDIES}

Now we analyze the time evolution of the SWE reference test (Figs. 3a-c). We use the nondimensional time $t^{\prime}=-\delta_{0} t$ and choose the $t_{a}^{\prime}=0.50, t_{b}^{\prime}=1.46$, and $t_{c}^{\prime}=2.98$ snapshots that represent three characteristic stages. Figure 3 a shows the $t^{\prime}=t_{a}^{\prime}$ early stage where vorticity patches are so sparse that the coupling between old vortices and a new updraft is uncommon. Figure $3 \mathrm{~b}$ shows the $t^{\prime}=t_{b}^{\prime}$ middle stage where some vortices are lucky enough to receive multiple updrafts and begin to interact with each other. The converging flow facilitates the merger process by reducing the vortices' spacing while conserving their absolute circulation. Figure $3 \mathrm{c}$ shows the $t^{\prime}=t_{c}^{\prime}$ later stage where the $17 \mathrm{~m} \mathrm{~s}^{-1}$ tropical storm strength is roughly reached (e.g., Fig. 2b). The strong

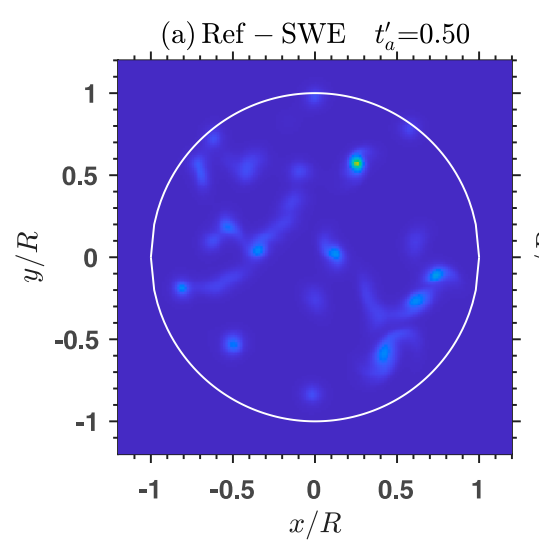

(d) Ref - WTGE $t_{a}^{\prime}=0.50$

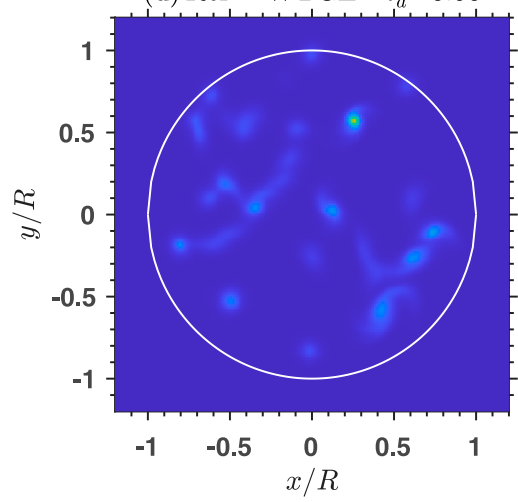

(g) Low - WTGE $t_{a}^{\prime}=0.50$

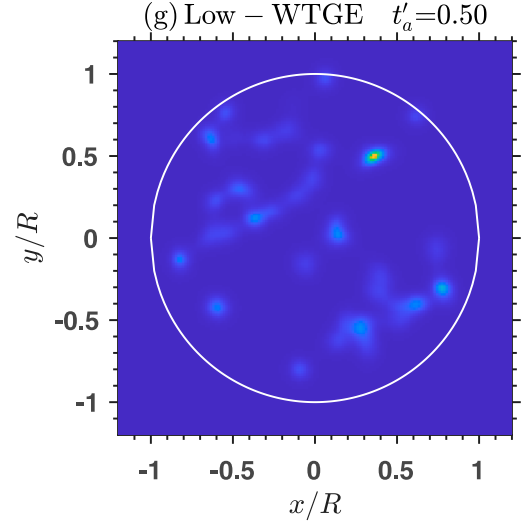

(b) Ref - SWE $\quad t_{b}^{\prime}=1.46$

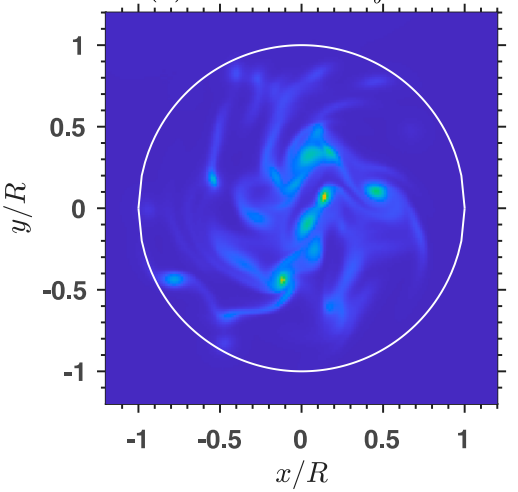

(e) Ref - WTGE $t_{b}^{\prime}=1.46$

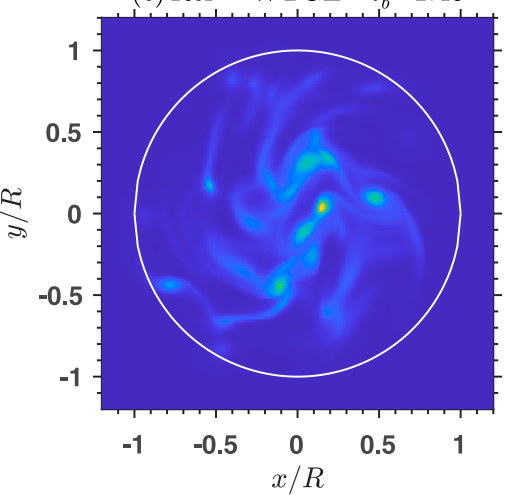

(h) Low - WTGE $\quad t_{b}^{\prime}=1.46$

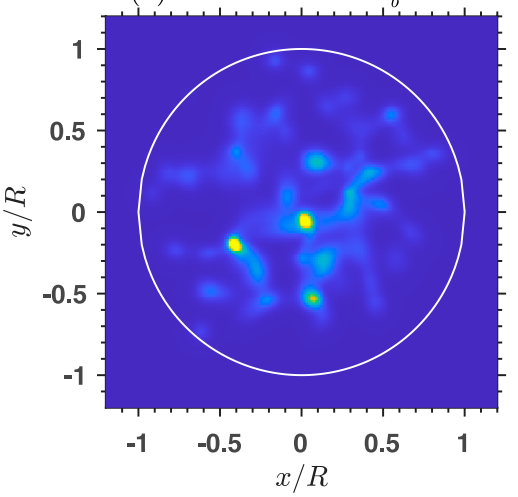

(c) Ref - SWE $\quad t_{c}^{\prime}=2.98$

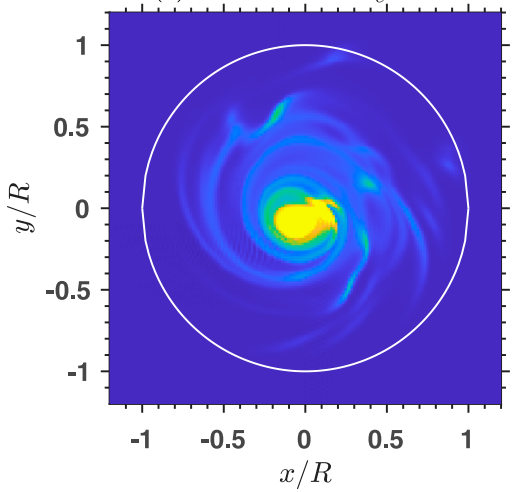

(f) Ref - WTGE $t_{c}^{\prime}=2.98$

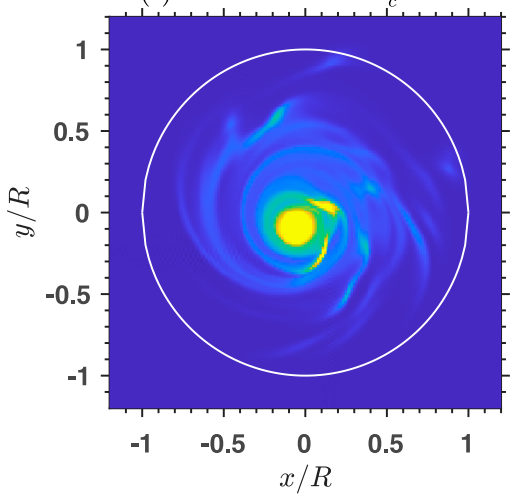

(i) Low - WTGE $\quad t_{c}^{\prime}=2.98$

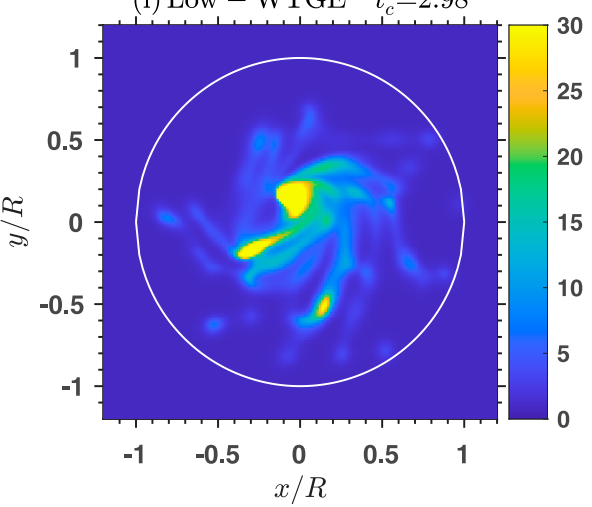

FIG. 3. An example of $\omega_{a} / f_{0}$ for (top) Ref-SWE, (middle) Ref-WTGE, and (bottom) Low-WTGE $\left(-\delta_{0} / f_{0} \times 10\right)$. For each test, only the ensemble-index- 1 run of the 20-member ensemble is shown. The convective seeding history of the three runs are identical. Columns show the(left) $t_{a}^{\prime}=-\delta_{0} t=0.50$ snapshot, (center) $t_{b}^{\prime}=1.46$, and (right) $t_{c}^{\prime}=2.98$. The white circle is the MCS boundary. The spatial coordinate has been normalized by $R$. 
vortices merge into a large major vortex, with the newly formed vorticity patches being rapidly distorted into filaments and wrapped around the core. The decrease of vorticity with radius produces an effective beta effect which migrates the small vortices toward the center, and helps the major vortex axisymmetrize itself (e.g., Terwey and Montgomery 2008; O'Neill et al. 2016). The merger is accompanied by elongated vorticity filaments that are produced by the strain in the mutual advection, as has been discussed by McWilliams (1990) and Dritschel and Waugh (1992). Though those filaments gradually wrap onto the vortex core, they are susceptible to strong dissipation when they are thin enough for diffusion to work. Some low-vorticity columns which lie between the filaments will also be wrapped inward and get mixed in the end. This reminds us of the finding by Fang and Zhang (2011) that most of the negative or weak vorticity patch is mixed with the large vorticity patch near the center of a major vortex, rather than being repelled out. The behavior beyond the tropical depression regime (maximum wind $>17 \mathrm{~m} \mathrm{~s}^{-1}$ ) is of less interest, because our simple convective scheme no longer works.

We compare the SWE test (named Ref-SWE) with WTGE test (named Ref-WTGE), as well as a low Coriolis parameter WTGE test (named Low-WTGE) that changes Coriolis parameter to $1 / 10$ of the reference value $\left(-\delta_{0} / f_{0} \times 10\right)$. Their convective seeding history of the ensemble-index- 1 run is set to be identical. Figure $2 \mathrm{~b}$ shows that both $\mathrm{Fr}$ and $-h^{\prime} / H$ in the SWE test are much smaller than 1 by $t^{\prime}=3$, so the WTG condition (5) is satisfied in the three snapshots. Figure 3 shows that the vorticity field difference between the Ref-SWE and Ref-WTGE is indeed small, even indiscernible at $t^{\prime}{ }_{a}=0.50$ and $t^{\prime}{ }_{b}=1.46$. As for the Low-WTGE, the inflow does push the vorticity patches toward the MCS center significantly, but the vorticity distribution is less axisymmetric and has less filamentation due to the much weaker rotational flow and therefore vortex interaction.

The traditional way to study the role of eddies in vortex formation or intensification is to diagnose the radial transport of vorticity by eddies (Hendricks et al. 2004; Montgomery et al.
2006), or check the energy cascade direction (e.g., Vallis et al. 1997; Wang et al. 2018). We propose a way to partly circumvent the turbulent process. The above simulations show that the vorticity produced by convection will sooner or later organize into a quasi-axisymmetric major vortex whose vorticity roughly monotonically decreases with radius. The latter is in agreement with the aircraft observation of a tropical depression by Middlebrooke (1988). Thus, the vorticity PDF can be related to the vorticity structure of the major vortex. Even if the monotonic and axisymmetric state is not completely reached, the vorticity PDF still qualitatively tells how compact the vortex is, which determines its ability to survive in a straining environment, as well as an upper bound of the maximum wind which signifies intensity. Note that the radial distribution of vorticity is not monotonic for a mature hurricane where deep convection at the eyewall makes the vorticity there larger than the quiescent eye (Schubert et al. 1999; Hendricks and Schubert 2009).

The PDF of the nondimensional absolute vorticity $x^{\prime}=$ $\ln \left(\omega_{a} / f_{0}\right)$ inside the MCS is defined as $\sigma$. The $\sigma$ of the three tests (Ref-SWE, Ref-WTGE, and Low-WTGE) is shown in Fig. 4. The PDF evolves from a purely low-vorticity-dominated state to a wide spectrum, with a tail extending to highvorticity region. It is not surprising that the PDF of Ref-SWE and Ref-WTGE collapse very well. However, the rough collapse of Ref-WTGE and Low-WTGE is intriguing, given their dramatically different flow field. This is explained in the next section with a simple vorticity PDF model. The small difference between the PDF of Ref-WTGE and Low-WTGE will be attributed to eddy mixing in section $6 \mathrm{a}$.

It is worth making a comparison with $3 \mathrm{D}$ turbulence cascade, where repetitive vortex stretching is also an important mechanism of scale reduction and vorticity amplification (Tennekes and Lumley 2018). The main difference in this model is the constraint of vortex orientation by the $2 \mathrm{D}$ domain (the stratified atmosphere) and the purely random prescription of the stretching (updraft) position. These make a statistical theory possible.
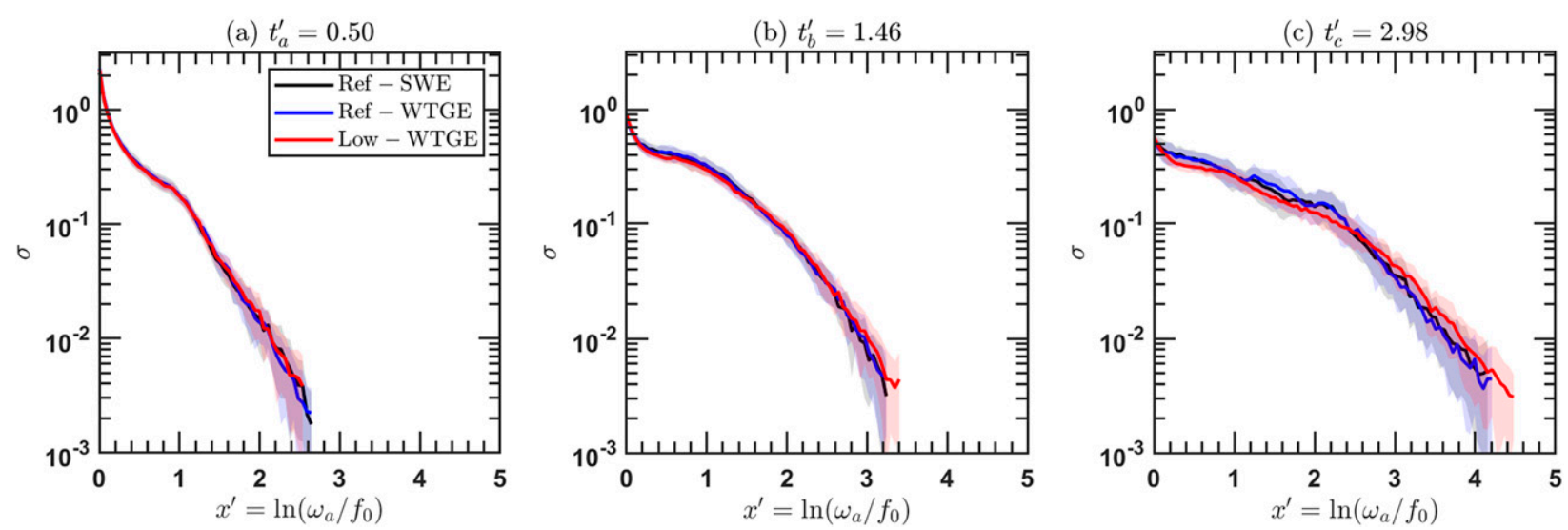

FIG. 4. The vorticity PDFs of Ref-SWE, Ref-WTGE, and Low-WTGE $\left(-\delta_{0} / f_{0} \times 10\right)$ for the reference test at (a) $t_{a}^{\prime}=0.50$, (b) $t_{b}^{\prime}=1.46$, and (c) $t_{c}^{\prime}=2.98$. The colored shadow denotes the \pm 1 standard deviation of the 20 -member ensemble of each test. The black, blue, and red lines are the ensemble average of Ref-SWE, Ref-WTGE, and Low-WTGE. The PDF from simulation is cutoff at the bin where the standard deviation is larger than the ensemble average. Only the $x^{\prime}=\ln \left(\omega_{a} / f_{0}\right)>0$ bins are shown. 


\section{A statistical theory of vorticity PDF}

\section{a. Motivation}

Within this setup, the vorticity PDF is largely governed by a random vorticity stretching process under the influence of Rayleigh drag and eddy mixing. Thanks to the validity of WTG approximation, potential vorticity reduces to absolute vorticity, so we do not need to consider the area change of vorticity patches during adiabatic motion. The negative vorticity produced by the inertially trapped compensating divergence near an updraft is also negligible. The eddy mixing, which does influence PDF via air column interactions, is a complicated process that is not included in this simple PDF model.

In section $5 b$, we model the PDF of the random stretching process as a discrete Markov chain. In section 5c, it is updated to a hybrid discrete-continuous PDF problem that includes Rayleigh drag.

\section{b. The random stretching problem}

The vorticity production in one convective event obeys (13). For multiple convective events, a new updraft may hit an existing vorticity patch and concentrate the vorticity there through stretching. As the strength of each updraft is fixed, the vorticity evolves by migrating on a set of discrete vorticity levels in power law:

$$
\omega_{a, m}=\omega_{a, 0}\left(1-\frac{\Delta h}{H}\right)^{m}, \quad m=0,1,2, \ldots ; \omega_{a, 0}=f_{0} .
$$

This concept is illustrated in Fig. 5. The parameter $-\delta_{0} \widetilde{\tau_{u}}$ does not appear because it does not influence the end state of a single updraft's geostrophic adjustment, and the interaction between updrafts is neglected due to $\sigma_{u} \ll 1$. Because the domain is much larger than the MCS, the descent is weak, so the negative vorticity production in the MCS due to vorticity squashing is neglected. As a new convection is seeded every $\Delta t$ time interval, the time level is also discrete:

$$
t_{n}=n \Delta t, \quad n=0,1,2, \ldots
$$

Let $\sigma_{m}^{n}$ be the fractional area of $\omega_{a, m}$ within the MCS at time level $n$. The quantity $\sigma_{m}^{n}$ can also be regarded as the discrete vorticity PDF, which satisfies

$$
\sum_{m=0}^{\infty} \sigma_{m}^{n}=1
$$

An updraft consumes the $\omega_{a, m}-1$ level area $\pi r_{u}^{2}(1-\Delta h / H)$, and the $\omega_{a, m}$ level gains area $\pi r_{u}^{2}$. Thus, the $\sigma_{m}^{n}$ evolution is essentially a discrete mapping of vorticity and time which is a linear Markov chain:

$$
\begin{gathered}
\sigma_{m}^{n}=\sigma_{m}^{n-1}(1-p)+\sigma_{m-1}^{n-1} \frac{r_{u}^{2}}{R^{2}}, \quad m=1,2, \ldots, M-1 \\
n=1,2, \ldots \\
\text { with } \quad p \equiv \frac{r_{u}^{2}}{R^{2}}\left(1-\frac{\Delta h}{H}\right)<1 .
\end{gathered}
$$

The $p$ is the probability for an air column in the MCS to get involved in each updraft event. The term $\sigma_{m}^{n-1}(1-p)$ in (18) denotes the loss of $m$ level vorticity due to the rise to $(m+1)$ level, and $\sigma_{m-1}^{n-1} r_{u}^{2} / R^{2}$ denotes the gain from $(m-1)$ level. Note that the property $(1-p)+r_{u}^{2} / R^{2}<1$ indicates net mass loss in an updraft. Such loss is compensated by the inflow across the MCS boundary which has a flux that depends on $\delta_{0}$ and is assumed to have zero relative vorticity due to the large domain effect. The lowest vorticity level $\sigma_{0}^{n}$ is determined by both the inflow refreshment rate and the migration to $\sigma_{1}^{n}$ level, with an initial value $\sigma_{0}^{0}=1$ :

$$
\sigma_{0}^{n}=(1-p) \sigma_{0}^{n-1}-\delta_{0} \Delta t
$$

The solution of $\sigma_{0}^{n}$ is

$$
\begin{aligned}
\sigma_{0}^{n}= & (1-p)^{n}\left(1+\frac{\delta_{0} \Delta t}{p}\right)-\frac{\delta_{0} \Delta t}{p} \\
= & {\left[1-\frac{r_{u}^{2}}{R^{2}}\left(1-\frac{\Delta h}{H}\right)\right]^{-t^{\prime} /\left[\left(r_{u}^{2} / R^{2}\right)(\Delta h / H)\right]}\left(1+\frac{\Delta h / H}{1-\Delta h / H}\right) } \\
& -\frac{\Delta h / H}{1-\Delta h / H} .
\end{aligned}
$$

Here we have used the nondimensional time $t^{\prime}=-\delta_{0} n \Delta t=$ $-\delta_{0} t$, and have used (12). For the reference value, $\delta_{0} \Delta t \approx$ -0.01 and $p \approx 0.03$.

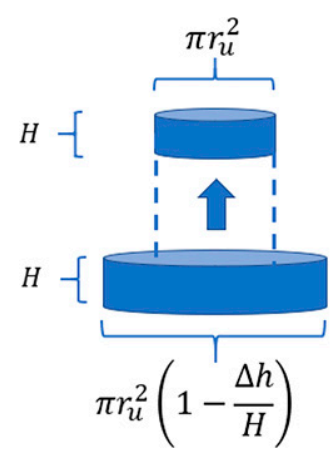

$$
\text { discrete levels of absolute vorticity } \omega_{a}=\omega+f_{0}
$$

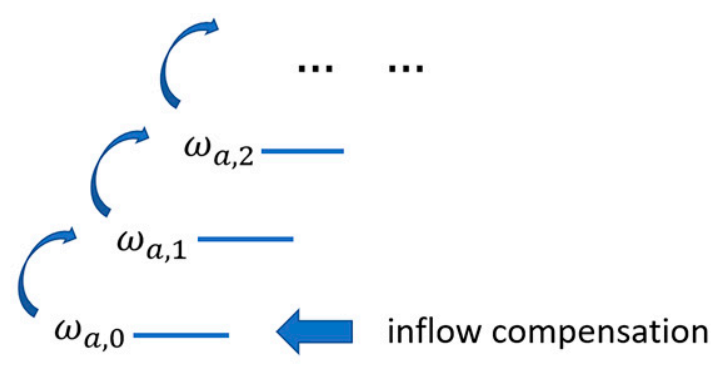

FIG. 5. A schematic diagram for the migration of air columns on the vorticity level system $\omega_{a, m}=\omega_{a, 0}(1-\Delta h / H)^{m}$ due to vorticity stretching, where $\omega_{a, 0}=f_{0}$. A column can only jump one level upward in a single updraft event. 
The change of $\sigma_{0}^{n}$ with $t^{\prime}$ is shown in Fig. 6a. Three features are noted.

- The quantity $r_{u}^{2} / R^{2}$ has little influence on $\sigma_{0}^{n}$ due to the smallness of $r_{u}^{2} / R^{2}$.

- For small $n$ (short time), $\sigma_{0}^{n} \approx(1-p)^{n}$, indicating that the compensating inflow is not important for early time when the MCS has not been occupied by vorticity patches.

- For $n \rightarrow \infty$ (long time), $\sigma_{0}^{n} \rightarrow-\delta_{0} \Delta t / p=(-\Delta h / H) /$ $(1-\Delta h / H)$, which is a constant that results from the balance between upward migration and inflow compensation and increases with the intermittency measure $-\Delta h / H$. An adjustment time scale $\tau_{\sigma_{0}}$ is obtained by linearizing (1 $p)^{n}$ to $1-n p$ and finding its zero-crossing time index $n=$ $1 / p$. As a verification, we find that $(1-p)^{1 / p}$ lies between 0.32 and 0.37 for $0<p<0.2$, which is quite robust. The expression of $\tau_{\sigma_{0}}$ is obtained with the help of (12) and (19): $\tau_{\sigma_{0}}=\Delta t / p=t /(n p)=\delta_{0}^{-1}(\Delta h / H) /(1-\Delta h / H)$, which also increases with the intermittency.

The solution of this discrete system is derived by analyzing the probability for a column to experience $m$ convective events by $t=$ $n \Delta t$ time, which turns out to be a weighted binomial distribution:

$$
\begin{aligned}
& \sigma_{m}^{n}= {\left[\left(\begin{array}{c}
n \\
m
\end{array}\right) p^{m}(1-p)^{n-m}+\sum_{k=1}^{n-m}\left(\begin{array}{c}
n-k \\
m
\end{array}\right)\right.} \\
&\left.p^{m}(1-p)^{(n-k)-m}\left(-\delta_{0} \Delta t\right)\right]\left(1-\frac{\Delta h}{H}\right)^{-m}, \\
& \text { for } \quad n \geq m>0, \quad \text { with } \quad\left(\begin{array}{c}
n \\
m
\end{array}\right)=\frac{n !}{m !(n-m) !} .
\end{aligned}
$$

It is easy to verify that (22) satisfies (18) and (21). As a comment, binomial and Poisson distributions have long been used to fit the probability of rare meteorological events (e.g.,
Thom 1957). Here $\sigma_{m}^{n}$ is not only contributed to by columns originally inside the MCS, but also those from outside that come in later at $t=\Delta t, 2 \Delta t, \ldots,(n-m) \Delta t$. For the native MCS columns, the probability to reach the $m$ level within $n$ updraft periods obeys a binomial distribution, as is shown in (22). Columns that come later at $t=k \Delta t$ time need to arrive at $m$ level within $(n-k) \Delta t$ time, as is represented by each term in the summation. The binomial distribution demonstrates that to migrate to the same level $m$, newer columns must be luckier than older ones because they have fewer discrete opportunities. To migrate to the $m$ level, the columns need to shrink their area by $(1-\Delta h / H)^{-m}$. Thus, all sources of contribution to $\sigma_{m}^{n}$ must be multiplied by this factor.

As $p$ is very small ( $p \approx 0.03$ for the reference test), the binomial distribution for large $n$ (sufficient for $n \geqslant 20$ or about $t^{\prime} \gtrsim-\delta_{0} \Delta t \times 20 \approx 0.2$ for the reference test) can be approximated as a Poisson distribution (Pishro-Nik 2014):

$$
\left(\begin{array}{c}
n \\
m
\end{array}\right) p^{m}(1-p)^{n-m} \approx \frac{(n p)^{m}}{m !} e^{-n p} .
$$

As $n p \sim t^{\prime} \ll 10$ in the regime of interest, the Poisson distribution element cannot be further approximated as a normal distribution. Substituting (23) into (22), using (12), and rearranging to better manifest the contribution from the original MCS columns and newcomers, we get an approximate $\sigma_{m}^{n}$ :

$$
\begin{aligned}
\sigma_{m}^{n} & \approx \frac{(n p)^{m} e^{-n p}}{m !}\left(1-\frac{\Delta h}{H}\right)^{-m}\left[1+\sum_{k=1}^{n-m}\left(1-\frac{k}{n}\right)^{m} e^{k p}\left(-\frac{\Delta h}{H} \frac{r_{u}^{2}}{R^{2}}\right)\right] \\
& \approx \frac{(n p)^{m} e^{-n p}}{m !}\left(1-\frac{\Delta h}{H}\right)^{-m}\left[1+t^{\prime} \int_{0}^{1}(1-s)^{m} e^{n p s} d s\right] .
\end{aligned}
$$

Here the sum has been approximated as an integral. It is valid when $d s \approx 1 / n=\left(r_{u}^{2} / R^{2}\right)(-\Delta h / H) / t^{\prime}$ is small and $m \ll n$, (a) $\sigma_{0}$

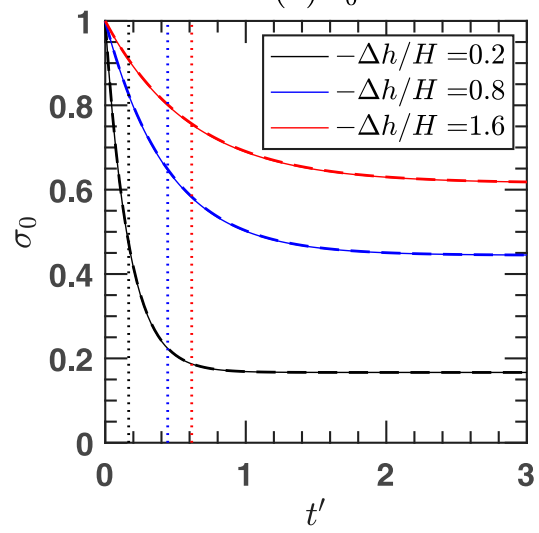

(b) $F_{0}$

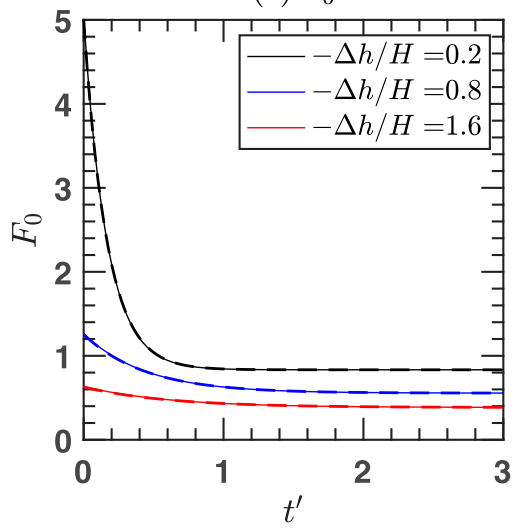

(c) $D_{i} \quad i=1,2,3,4$

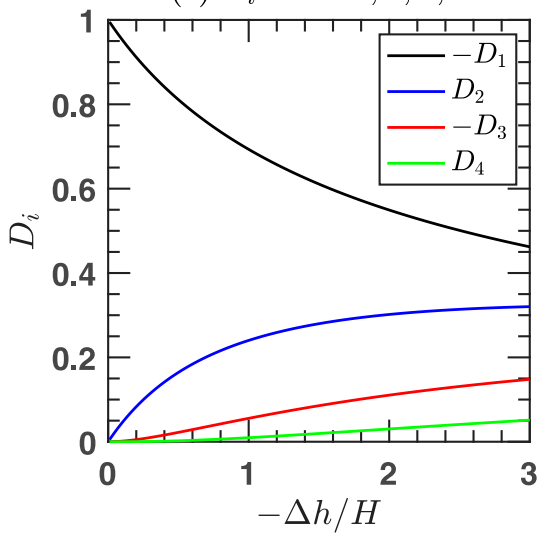

FIG. 6. (a) The evolution of the $\omega_{a}=f_{0}$ basic-level PDF value $\sigma_{0}$ with nondimensional time $t^{\prime}=-\delta_{0} t$ for $-\Delta h / H=0.2,0.8$, and 1.6 , denoted by the black, blue, and red lines, respectively. The solid lines are for $r_{u}=8 \sqrt{2} \mathrm{~km}$ (reference value), and the dashed lines are for $r_{u}=4 \sqrt{2} \mathrm{~km}$. They collapse well. The dotted lines are the nondimensional adjustment time scale $-\delta_{0} \tau_{\sigma_{0}}=(-\Delta h / H) /(1-\Delta h / H)$ for the three different $-\Delta h / H$. (b) As in (a), but for the probability current $F_{0}$, which is the $F$ at $x^{\prime}=\Delta x^{\prime} / 2$. (c) The dependence of the PDF equation coefficients $D_{1}, D_{2}, D_{3}$, and $D_{4}$ on $-\Delta h / H$, denoted as the black, blue, red, and green line, respectively. As $D_{1}$ and $D_{3}$ are negative, we plot $-D_{1}$ and $-D_{3}$ to ease visualization. 
therefore applicable to our $r_{u} / R \ll 1$ case at a not too small $t^{\prime}$. Because the Poisson distribution parameter is $n p=t^{\prime}(1-$ $\Delta h / H) /(-\Delta h / H)$, the only physical parameter involved in this integral is $-\Delta h / H$. Why is $r_{u} / R$ unimportant? Physically, for fixed $\delta_{0}$ and $-\Delta h / H$, a wider updraft is equivalent to a bunch of narrower updrafts which are seeded at the same time and cannot overlap with each other. It is this nonoverlap requirement that causes the difference. However, the chance of overlap in seeding a bunch of narrower updrafts independently is already very small for $r_{u} / R \ll 1$, so the difference is tiny.

This analytical solution additionally tells how different ages of columns constitute a certain level of vorticity. This might be useful for studying tracer transport by a tropical depression. As $\sigma_{m}^{n}$ is linked to the radial distribution of vorticity within the major vortex at the later stage, (24) also qualitatively tells the age composition at different radial positions. Figure 7 shows the contribution to $\sigma_{m}^{n}$ at $m=2$ and 5 levels from the columns entering at $t_{k}=k \Delta t$ (only $k \geq 1$ is shown), with the reference test parameter. This fraction $\mu_{m}(k, n)$ is

$$
\begin{aligned}
\mu_{m}(k, n) \equiv & \left(1-\frac{k}{n}\right)^{m} e^{k p}\left(-\frac{\Delta h}{H} \frac{r_{u}^{2}}{R^{2}}\right) \\
& {\left[1+\sum_{k=1}^{n-m}\left(1-\frac{k}{n}\right)^{m} e^{k p}\left(-\frac{\Delta h}{H} \frac{r_{u}^{2}}{R^{2}}\right)\right]^{-1} . }
\end{aligned}
$$

A "maximum-contribution age" always exists for a large $n$, because many older air columns have migrated to higher levels and the younger air columns do not have much chance to reach that level. This age is younger for a lower $m$, which is easier to reach.

\section{c. The hybrid discrete-continuous PDF}

The "vorticity-equivalent top-hat" and therefore the discrete vorticity level system is only a mathematical approximation. Each updraft can produce a continuous range of $\omega_{a}$, and the PDF increment at a certain $\omega_{a}$ can be contributed by a continuous range of $\omega_{a}$ below it. Meanwhile, the discrete base level $\omega_{a}=f_{0}$, which depends on the balance of convective occupation and inflow compensation, is well defined. Thus, we propose a hybrid view that retains the $\omega_{a}=f_{0}$ level fractional area $\sigma_{0}$ as a discrete one, and let the larger $\omega_{a}$ be a continuous distribution $\sigma_{c}$.

As a standard technique, the original discrete vorticity levels can be viewed as a discrete sampling, or a finite-difference approximation to a continuous PDF (Pope 2001). Because the original discrete set of levels is a power law that is uniform on a logarithmic coordinate, we introduce the continuous levels as $x^{\prime}=\ln \left(\omega_{a} / f_{0}\right)$, which has been used in Fig. 4. In comparison, the original discrete levels are located at $x_{m}^{\prime}=$ $\ln \left(\omega_{a, m} / f_{0}\right)=m$, with an interval of $\Delta x^{\prime}=\ln (1-\Delta h / H)$. We then introduce the continuous PDF $\sigma_{c}$, which approximately represents the homogenization of $\sigma_{m}^{n}$ within $x_{m}^{\prime} \pm(1 / 2) \Delta x^{\prime}$ :

$$
\sigma_{c}\left(x_{m}^{\prime}, t^{\prime}\right) \approx \frac{\sigma_{m}^{n}}{\Delta x^{\prime}}, \quad m=1,2,3 \ldots
$$

The governing equation of $\sigma_{c}$, also called Kramers-Moyal equation (Pope 2001), is derived in the supplemental material.

The hybrid PDF is not only more physically realistic, but it is also straightforward to incorporate Rayleigh drag. It is hard to add drag to the Markov chain model, because the vorticity levels change continuously due to the drag. As a result, new vorticity levels are produced every $\Delta t$ and the number of levels blows up.

The PDF of the discrete part, $\sigma_{0}$, is an updated version of (21). After replacing $n$ by $t^{\prime} / \Delta t^{\prime}\left(t^{\prime}=-\delta_{0} t\right.$ and $\left.\Delta t^{\prime}=-\delta_{0} \Delta t\right)$ to make it continuous in time, we get

$$
\sigma_{0}\left(t^{\prime}\right)=(1-p)^{t^{\prime} / \Delta t^{\prime}}\left(1-\frac{\Delta t^{\prime}}{p}\right)+\frac{\Delta t^{\prime}}{p} .
$$

How do the discrete and continuous part match with each other? In view of finite difference, the discrete "grid cell"
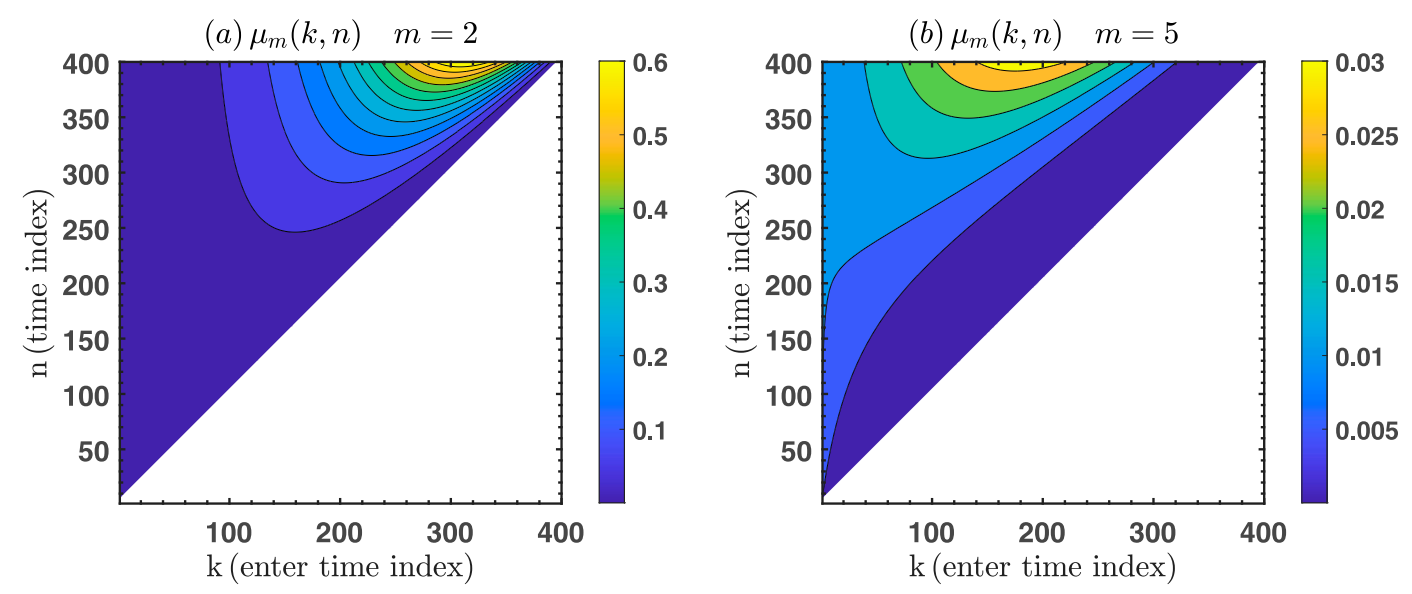

FIG. 7. The fractional contribution $\mu_{k}$ to $\sigma_{m}^{n}$ for different ages of air columns predicted by the theory, using the reference test parameter. The horizontal axis is the time index $k$ on which the column enters the MCS, and the vertical axis is the time index $n$. The blank region denotes $\mu_{k}=0$. (a) The $m=2$ vorticity level and (b) the $m=5$ level. Note that both $n$ and $k$ are positive integers. Only $k \geq 1$ columns, which are not originally inside the MCS are plotted. 
spans $0 \pm \Delta x^{\prime} / 2$. The domain of continuous PDF is heuristically set as $x^{\prime} \in\left(\Delta x^{\prime} / 2,+\infty\right)$. Inspired by Sukhatme and Young (2011) in treating the vapor PDF equation of an advection-condensation model, the $\sigma$ in this domain is set to satisfy the normalization condition: $\int_{\Delta x^{\prime} / 2}^{\infty} \sigma_{c} d x^{\prime}=1-\sigma_{0}\left(t^{\prime}\right)$ which corresponds to a flux boundary condition at the small $x^{\prime}$ end $\left(x^{\prime}=\Delta x^{\prime} / 2\right)$, as is derived in the supplemental material.

The deterministic problem of the continuous part $\sigma_{c}$ which uses the nondimensional time $t^{\prime}=-\delta_{0} t$ is

$$
\begin{gathered}
\frac{\partial \sigma_{c}}{\partial t^{\prime}}+\frac{\partial F}{\partial x^{\prime}}=-\sigma_{c} \\
\text { with } F=-D_{1} \sigma_{c}-\sum_{i=1}^{3} D_{i+1} \frac{\partial^{i} \sigma_{c}}{\partial x^{\prime i}}+\frac{\sigma_{c}}{\delta_{0} \tau_{d}}\left(1-e^{-x^{\prime}}\right) \\
\qquad x^{\prime} \in\left(\Delta x^{\prime} / 2,+\infty\right) \\
\left.F_{0} \equiv F\right|_{x^{\prime}=\Delta x^{\prime} / 2} \\
=-\frac{d \sigma_{0}}{d t^{\prime}}+\left(1-\sigma_{0}\right),\left.\quad \sigma_{c} t\right|_{x^{\prime} \rightarrow \infty}=0,\left.\quad \sigma_{c}\right|_{t^{\prime}=0}=0
\end{gathered}
$$

Here $F$ is the probability current, and $F_{0}$ is the $F$ at $x^{\prime}=\Delta x^{\prime} / 2$. The $F$ includes the vorticity migration process and the Rayleigh drag. The derivative terms come from a Taylor expansion that represents the nonlocal migration nature of the Markov chain (convective intermittency). The drift, diffusion, dispersion, and hyperdiffusion coefficients are

$$
D_{i}=\frac{(-1)^{i-1}}{i !} \frac{H}{\Delta h}\left[\ln \left(1-\frac{\Delta h}{H}\right)\right]^{i}, \quad i=1,2,3,4 .
$$

Here $D_{1}<0, D_{2}>0, D_{3}<0, D_{4}>0$. $F$ can include higher derivative terms, but we found that truncation to the $D_{4}$ term yields sufficient accuracy for our $-\Delta h / H \sim O(1)$. The full PDF expression is

$$
\sigma\left(x^{\prime}, t^{\prime}\right)=\sigma_{0}\left(t^{\prime}\right) \Theta\left(x^{\prime}\right)+\sigma_{c}\left(x^{\prime}, t^{\prime}\right),
$$

where $\Theta$ denotes the Dirac delta function. Equations (27), (28), (30), (31), and (32) form a closed problem that can only be solved numerically in general.

Of the six nondimensional parameters that control the whole WTGE problem, the PDF problem is only controlled by two: $\Delta h / H$ on convective intermittency and $\delta_{0} \tau_{d}$ on drag. The updraft size $r_{u}^{2} / R^{2}$ does exist in the expression of $\sigma_{0}\left(t^{\prime}\right)$ but has tiny influence, as explained in section 5b. Physically, the $\widetilde{\tau_{u}}$ does not appear because it does not directly influence the end state of the vorticity spinup by an individual convection. The nondimensional independent variables show that the solution is self-similar to $f_{0}$ and $\delta_{0}$. A larger $f_{0}$ (tropical cyclogenesis at higher latitude) systematically raises the magnitude of vorticity. A larger $\delta_{0}$ magnitude (larger MCS-averaged updraft mass flux) simply accelerates the PDF evolution. In fact, the problem can be extended to an unsteady $\delta_{0}(t)$ by replacing the temporal coordinate $t^{\prime}=-\delta_{0} t$ with a stretched one: $t^{\prime}=-\int_{0}^{t} \delta_{0}\left(t^{\prime \prime}\right) d t^{\prime \prime}$, which is the accumulated convergence of the system (MCS). This is used in section 7 where the theory is applied to interpret the 3D cloud-permitting simulation. For the Markov chain model in section 5 b where Rayleigh drag is not included, the only change is regarding $\Delta t$ as a function of $t$ in $t_{n}=$ $n \Delta t$.

The " $\sigma_{c}$ " term on the right-hand side (rhs) of (28) is a linear damping factor on the PDF that denotes the area shrinking due to flow convergence. The magnitude of the drift coefficient $\left|D_{1}\right|$ decreases with increasing $-\Delta h / H$, but the magnitude of the higher-order coefficients increases with $-\Delta h / H$, as is shown in Fig. 6c. This leads to a flatter tail on the PDF. It indicates that a more intermittent convective mode leads to more high-vorticity air columns. The Rayleigh drag is essentially an "antiadvection" that pushes the PDF toward a lowvorticity region and accumulates there. It is only significant for a long enough time: $t^{\prime} \gtrsim-\delta_{0} \tau_{d}$.

Figures $6 \mathrm{a}$ and $6 \mathrm{~b}$ show the time evolution of $\sigma_{0}$ and $F_{0}$ for different $-\Delta h / H$. The entering probability flux $F_{0}$ is largest at the beginning, because every updraft can turn a piece of the vast $\omega_{a}=$ $f_{0}$ region into $\omega_{a}>f_{0}$. It decreases rapidly to $1-\sigma_{0}$ where the occupation by updraft is balanced by the supply of $\omega_{a}=f_{0}$ area by the inflow. The adjustment time scale of $F_{0}$ is $\tau_{\sigma_{0}}$, which is identical to that of $\sigma_{0}$. The less intermittent the updraft is, the larger $F_{0}$ is at the early stage due to the rapid occupation, and the faster $F_{0}$ falls back to 1 .

\section{d. The analytical solution in the uniform updraft limit}

For $-\Delta h / H \rightarrow 0$ (very weak but frequent convection), the hybrid PDF problem renders an analytical solution. Here, $D_{1} \rightarrow-1, D_{2}, D_{3}, D_{4}$ vanish, and $F_{0}$ contains a pulse at the beginning. The quantity $\sigma_{0}$ drops to 0 very fast due the rapid occupation by convection. Thus, the discrete part of the PDF occupies an infinitesimal space on $x^{\prime}$, and the hybrid problem is solely controlled by the continuous part which spans $x^{\prime} \in(0,+\infty)$. This situation is equivalent to a uniform mass sink in the MCS, and the MCS dynamics is just the larger-scale version of the single updraft vorticity spinup process introduced in appendix B.

Thus, the solution of vorticity spinup by a single convection in physical space can serve as a benchmark for the PDF equation. Through the derivation in the supplemental material, we get

$$
\sigma\left(x^{\prime}, t^{\prime}\right)=\sigma_{c}\left(x^{\prime}, t^{\prime}\right)= \begin{cases}{\left[\Theta\left(t^{\prime}\right)+1\right] e^{-x^{\prime}},} & x^{\prime} \leq t^{\prime}, \\ 0, & x^{\prime}>t^{\prime}\end{cases}
$$

The PDF is not monotonic: it consists of a $x^{\prime}=t^{\prime}$ peak followed by an exponentially decaying slope, without a highvorticity "tail" (e.g., Fig. 8a). The delta function part in PDF corresponds to the solid body vortex core. It consists of the air columns initially inside the MCS. Now, we link the vorticity PDF with its physical distribution. The vorticity is resorted, with the lowest vorticity at the rim of the MCS and the highest vorticity at its core. Each infinitesimally thin ring in physical space corresponds to an infinitesimally small bin in the PDF:

$$
\int_{0}^{r} \frac{2 \pi r}{\pi R^{2}} d r=\int_{x^{\prime}}^{+\infty} \sigma d x^{\prime}
$$

This recovers the physical space solution that consists of a solid body rotation core and a decaying skirt: 

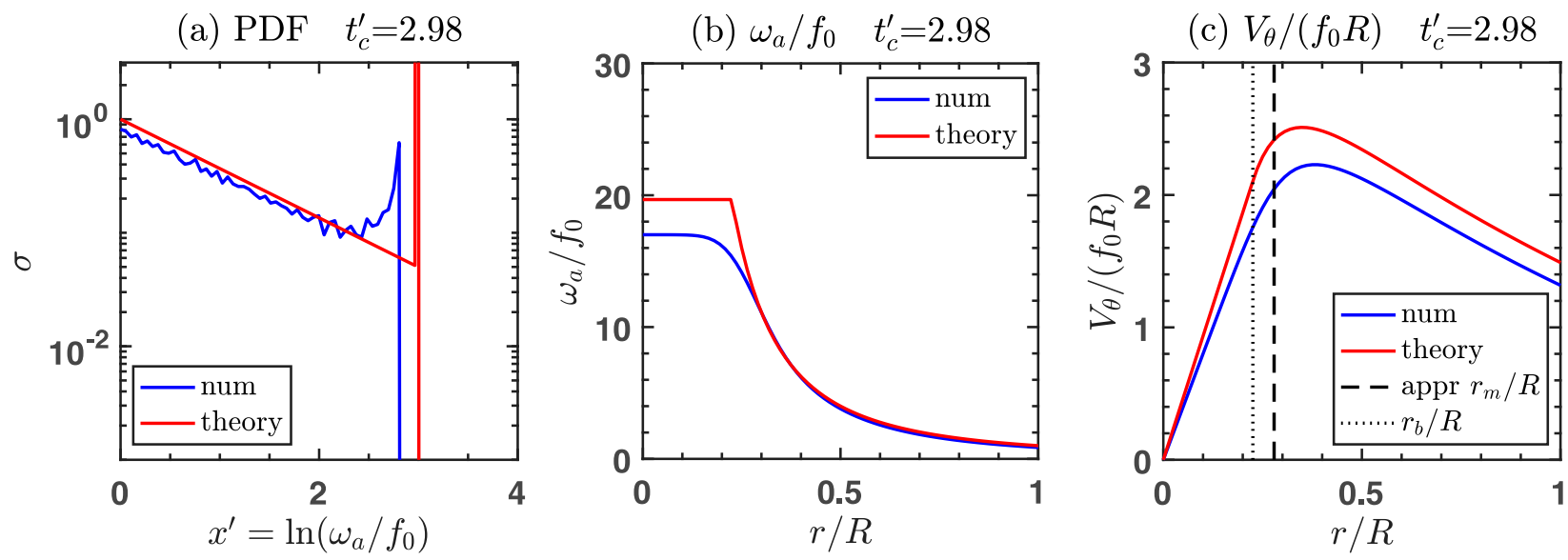

FIG. 8. The $-\Delta h / H \rightarrow 0$ axisymmetric problem. (a) The blue line is the vorticity PDF diagnosed from the gridpoint data of the barotropic simulation with the reference value Re, and the red line is the direct analytical solution of the PDF shown in (33). (b) A radial cross section of the vorticity field at $t^{\prime}{ }_{c}=2.98$. The blue line is the result of barotropic simulation, and the red line is the analytical solution. The difference is mainly due to viscosity and the finite-domain effect. (c) As in (b), but for the local Rossby number: $V_{\theta} /\left(f_{0} R\right)$. An approximate expression of the radius of maximum wind $r_{m} \approx r_{b}\left[1+\left(1-e^{-t^{\prime}}\right) / 4\right]$ and the vortex core radius $r_{b}$, both normalized by $R$, are additionally plotted as the dashed black line and the dotted black line, respectively.

$$
\omega_{a}= \begin{cases}f_{0} e^{t^{\prime}}, & \frac{r}{R}<e^{-t^{\prime} / 2}, \\ f_{0}\left(\frac{r}{R}\right)^{-2}, & \frac{r}{R} \geq e^{-t^{\prime} / 2} .\end{cases}
$$

This is equivalent to (B1), after replacing $\widetilde{r_{u}}$ by $R,-\delta_{u} \widetilde{T_{u}}$ by $t^{\prime}=-\delta_{0} t$, and $\omega_{a, 0}$ by $f_{0}$. Let $r_{b}=\operatorname{Re}^{-t^{\prime} / 2}$ be the radius of the solid-body rotation core. The finite domain effect reduces the peak vorticity in the numerical solution and the diffusion smears the vortex core rim, but the theory is otherwise very similar (Figs. 8b,c).

The tangential velocity $V_{\theta}$ is obtained by integrating (35) radially. Upon being rescaled by $f_{0} R$, it is viewed as a local Rossby number:

$$
\frac{V_{\theta}}{f_{0} R}= \begin{cases}\left(e^{t^{\prime}}-1\right) \frac{r}{2 R}, & \frac{r}{R}<e^{-t^{\prime} / 2}, \\ \frac{1}{2}\left(\frac{r}{R}\right)^{-1}\left(1+t^{\prime}\right)+\left(\frac{r}{R}\right)^{-1} \ln \left(\frac{r}{R}\right)-\frac{r}{2 R}, & e^{-t^{\prime} / 2} \leq \frac{r}{R} \leq 1, \\ \frac{t^{\prime}}{2} \frac{R}{r}, & \frac{r}{R}>1 .\end{cases}
$$

Equation (36) is also the result of angular momentum conservation, which is approximately valid during the spinup of the lower-free-tropospheric flow. Unlike Rankine vortex whose radius of maximum azimuthal mean wind is located at the boundary of its solid body core, that of this model (defined as $r=r_{m}$ ) is located outside of the solid body core $r=r_{b}$, and the maximum azimuthal mean azimuthal wind $\overline{V_{m}}$ is always a bit larger than the core boundary velocity $V_{b}$ (e.g., Figs. 8b,c). Though $r_{m}$ and $\overline{V_{m}}$ do not have analytical expression, $r_{b}$ and $V_{b}$ do:

$$
\frac{V_{b}}{f_{0} R}=\sinh \left(\frac{t^{\prime}}{2}\right) \quad \text { at } r_{b} / R=e^{-t^{\prime} / 2}
$$

A Taylor expansion of $V_{\theta}$ around $r_{b}$ shows that $r_{m} \approx r_{b}\left[1+\left(1-e^{-t^{\prime}}\right) / 4\right]>r_{b}$, and $\overline{V_{m}}>V_{b}$. In section $6 \mathrm{c}$, we will show that $V_{b}$ is a good approximation of $\overline{V_{m}}$ for this axisymmetric case and is useful for understanding the evolution of the maximum wind in other cases.

\section{Validation and sensitivity tests}

In this section, we compare the PDF theory with WTGE numerical simulation, and discuss how the convective parameters influence the compactness and intensity of the idealized tropical depression. We primarily perform six tests:

- EXP-a: The reference test (the Ref-WTGE).

- EXP-b: The $-\widetilde{\Delta h} / H \times 1 / 8$ (or $-\Delta h / H \times 1 / 8$ ) test. It is performed by making the convective lifetime $-\delta_{0} \widetilde{T}_{u}$ be $1 / 8$ of the reference value and holding the nondimensional updraft mass sink rate $\widetilde{Q_{m}} /\left(-\delta_{0} H\right)=(\widetilde{\Delta h} / H) /\left(-\delta_{0} \widetilde{T_{u}}\right)$. We will see $-\delta_{0} \widetilde{T}_{u}$ is not a sensitive parameter, so letting it change together with $-\widetilde{\Delta h} / H$ should not add meaningful complexity. 
- EXP-c: The $\tau_{d}$ drag test, with $-\delta_{0} \tau_{d}=2.0$, equivalent to a damping time scale of $\tau_{d} \approx 2$ days. A realistic $\tau_{d}$ based on the estimation of Montgomery et al. (2001) for a weak hurricane is $\sim 3$ days. We use this exaggeratedly short $\tau_{d}$ to theoretically understand the influence of strong drag, and to test the PDF model as well.

- EXP-d: The $\widetilde{r_{u}} / R \times 2$ test, performed by making $\widetilde{r_{u}} \times 2$. With the nondimensional argument in appendix A, it can also be regarded as halving the MCS radius.

- EXP-e: The $-\delta_{0} \widetilde{T_{u}} \times 1 / 4$ test, performed by making the updraft mass sink rate $\overline{Q_{m}} \times 4$.

- EXP-f: The $\operatorname{Re} \times 1 / 2$ test, performed by doubling $\nu$.

These, together with the $-\delta_{0} / f_{0} \times 10$ test (Low-WTGE) introduced in section $4 b(2)$, cover all the six nondimensional parameters.

\section{a. Vorticity PDF}

Figure 9 shows the PDF predicted by the analytical solution of the Markov chain in (24) and the numerical solution of the continuous part of the hybrid theory $\left(\sigma_{c}\right)$ against the WTGE simulation, at $t_{b}^{\prime}=1.46$ and $t_{c}^{\prime}=2.98$ (the middle and later stage). First, for all the tests without Rayleigh drag, the agreement with the hybrid PDF theory and the Markov chain is good. Second, the agreement of the hybrid PDF theory with the barotropic simulation is good, except that there is overestimation at the large $x^{\prime}$ region and underestimation at the middle $x^{\prime}$ region. Such deviation is more significant for $t_{c}^{\prime}=$ 2.98 than $t_{b}^{\prime}=1.46$, and is weaker for the $\tau_{d}$ test.

We judge that turbulent mixing is responsible for a substantial portion of the deviation, because the $-\delta_{0} / f_{0} \times 10$ test has weaker turbulent mixing and is closer to the theory (Fig. 4). The eddies not only help concentrate positive vorticity at the core, but also mix the low- and high-vorticity columns into the middle range. The mixing looks like an "antidiffusion" on PDF (Pope 2001). This also explains why the $\tau_{d}$ test has a lower deviation: the turbulence is damped by drag and therefore cannot produce small-scale filaments effectively.

Only the PDF of the $-\Delta h / H$ and $\tau_{d}$ tests are significantly different from the reference test. This agrees with the
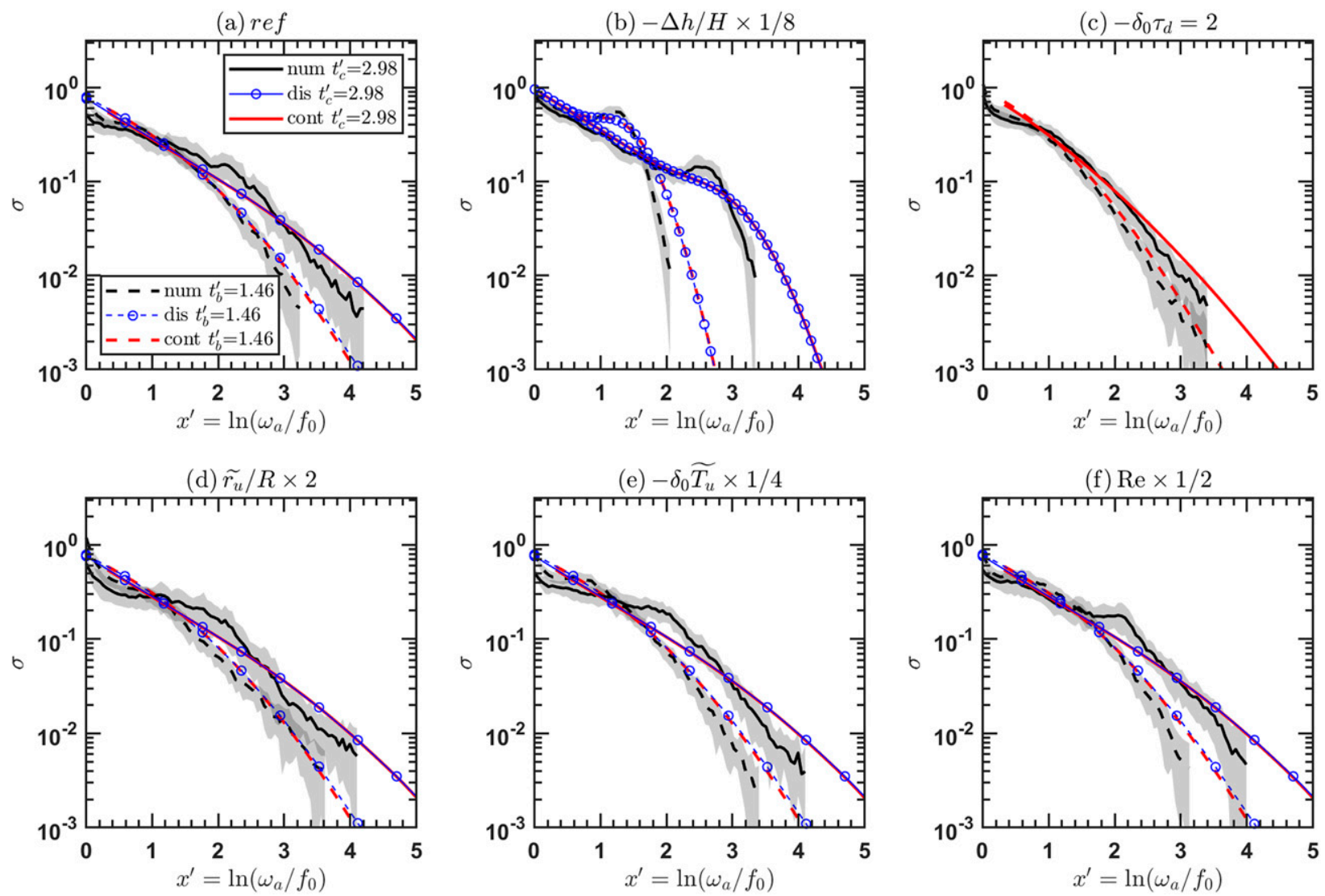

FIG. 9. The vorticity PDF of the six WTGE tests at $t_{b}^{\prime}=1.46$ and $t_{c}^{\prime}=2.98$, of (a) the reference test, (b) the $-\Delta h / H \times 1 / 8$ (equivalent to $-\widetilde{\Delta h} / H \times 1 / 8)$ and $-\delta_{0} \widetilde{T}_{u} \times 1 / 8$ test, (c) the $-\delta_{0} \tau_{d}=2$ test, (d) the $\widetilde{r_{u}} / R \times 2$ test, (e) the $-\delta_{0} \widetilde{T}_{u} \times 1 / 4$ test, and (f) the Re $\times 1 / 2$ test. The gray shadow is the \pm 1 standard deviation of the ensemble runs. The black line is the ensemble average in each test. The blue circle line is the analytical solution of the discrete PDF shown in the first line of (24), which uses the time index $n$ closest to the inquired time. The red line is the numerical solution of the continuous part $\left(\sigma_{c}\right)$ of the hybrid PDF problem. The discrete PDF model of the $\tau_{d}$ test is unavailable. For all cases, the dashed and solid lines denote $t_{b}^{\prime}=1.46$ and $t_{c}^{\prime}=2.98$ case, respectively. The PDF from the simulations (the black line and the shading) is cutoff at the bin where the standard deviation is larger than the average. Only $x^{\prime}=\ln \left(\omega_{a} / f_{0}\right)>0$ bins are shown. 
theoretical prediction that the PDF problem is only controlled by $-\Delta h / H$ and $-\delta_{0} \tau_{d}$ to the lowest order. The vorticity PDF is less spread when $-\Delta h / H$ is small. The $\tau_{d}$ test shows that Rayleigh drag damps the mid- and high-vorticity part of the spectrum significantly. The PDF is related to the spatial structure of the major vortex (Fig. 10). By $t_{c}^{\prime}=2.98$, a major vortex has been established, though some asymmetric structure still exists due to the unfinished merger process. In the $-\Delta h / H \times 1 / 8$ test, the major vortex is more axisymmetric but less compact. In the $\tau_{d}$ test, the major vortex is small and weak compared to its filaments due to the weaker vorticity magnitude and therefore weaker vortex interaction.

There is some dispersion of the PDF ensemble, especially at the large vorticity range where some bins are empty but some are not. Such uncertainty is due to the spatiotemporal discrete nature of convective events. The $-\Delta h / H=-\delta_{0} \tau_{\text {rev }} \rightarrow 0$ case converges to fully deterministic due to the infinitely short convective revisit time.

The PDF is insensitive to viscosity for the Re range we use. This is because the two main dissipation processes in our model-the flattening of a vortex patch by diffusion after a stretching event and the eddy mixing - are smallscale processes that are sufficiently separated from the scale of vortex interaction. A larger diffusivity increases the dissipative scale, so the dissipative effect is largely unchanged.

\section{b. Asymmetry and monotonicity}

The relationship between the PDF and the vorticity radial distribution is closer when the vorticity is more axisymmetric and more radially monotonic. We define a nonaxisymmetric and nonmonotonic index (NAMI) to quantify these two factors. The vortex center is defined as the maximum point of a Gaussian filtered vorticity field (with a filter length scale of $0.3 R$ ). This treatment loosely considers both the geometrical center and the strongest eddy's center. A larger filter length adds weight to the former. Based on this, we define the radial profile of the azimuthal-average vorticity as $\bar{\omega}$. We then define $\omega_{\text {res }}$ as the axisymmetric and monotonic vorticity field obtained from resorting all the simulated vorticity grid points in a circle with a radius of $R$ whose center is the vortex center. The air column (grid point) with the highest vorticity is put in the center and the lower vorticity columns are wrapped around it. The NAMI is defined as a normalized quadratic difference between $\bar{\omega}$ and $\omega_{\text {res: }}$ :

$$
\mathrm{NAMI} \equiv \frac{\int_{0}^{1}\left(\bar{\omega}-\omega_{\mathrm{res}}\right)^{2} d(r / R)}{\int_{0}^{1} \bar{\omega}^{2} d(r / R)},
$$

where $r$ denotes the distance from the vortex center. To give more weight to the central region, the integral in (38) is not weighted by $r$. The NAMI is zero when the vortex is perfectly

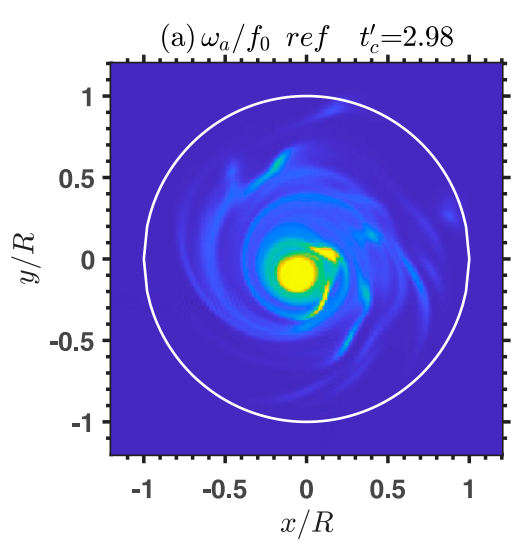

(d) $\widetilde{r_{u}} / R \times 2 \quad t_{c}^{\prime}=2.98$

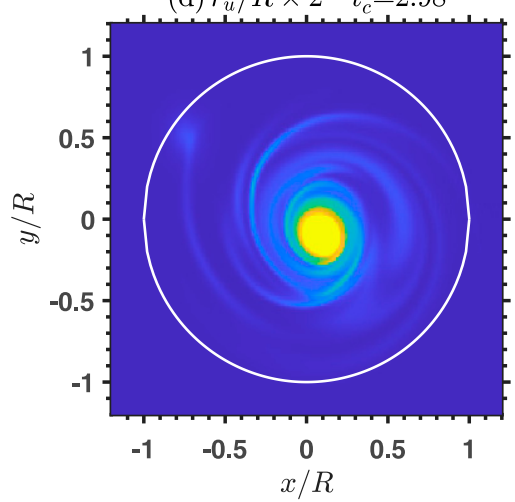

(b) $-\Delta h / H \times 1 / 8 \quad t_{c}^{\prime}=2.98$

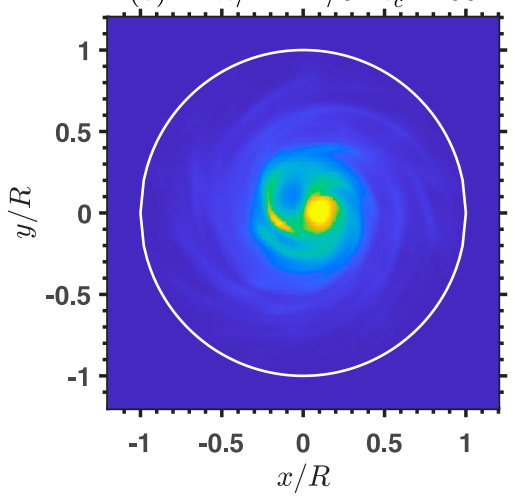

(e) $-\delta_{0} \widetilde{T_{u}} \times 1 / 4 \quad t_{c}^{\prime}=2.98$

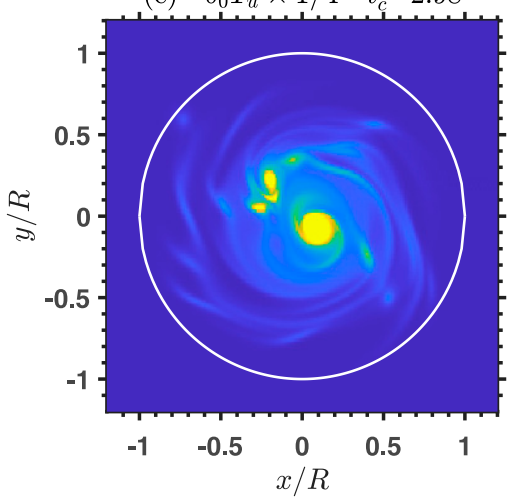

(c) $-\delta_{0} \tau_{d}=2 \quad t_{c}^{\prime}=2.98$

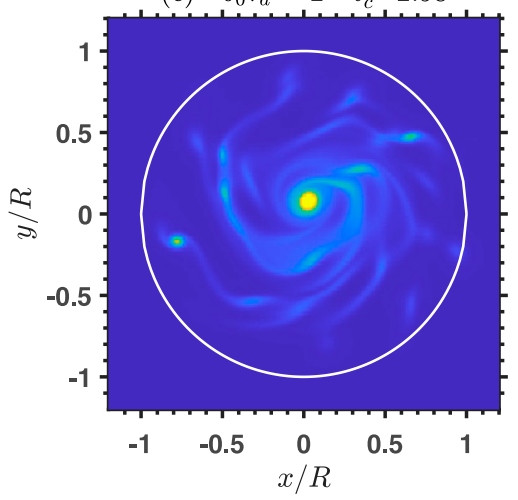

(f) $\operatorname{Re} \times 1 / 2 \quad t_{c}^{\prime}=2.98$

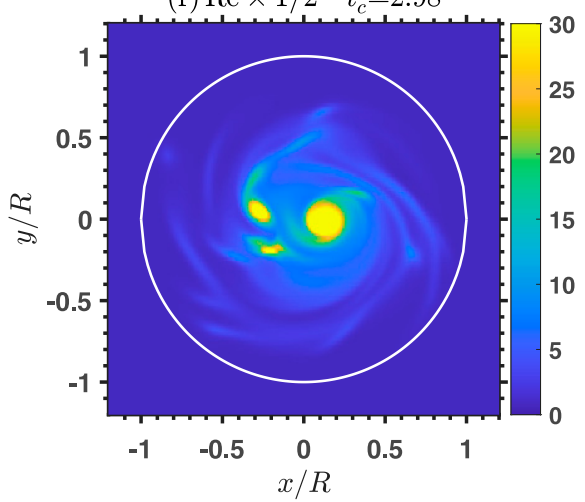

FIG. 10. An example of the $\omega_{a} / f_{0}$ snapshot of the same six tests as in Fig. 9 at $t^{\prime}{ }_{c}=2.98$. The white circle is the MCS boundary.

Of the 20 runs in each ensemble, only the ensemble-index- 1 run is shown. 
axisymmetric and monotonic. The procedure is analogous to calculating the available potential energy of a stratified flow (Winters et al. 1995; Vallis 2017). Apparently, a higher $-\delta_{0} / f_{0}$ yields a lower NAMI due to the lack of eddies that could axisymmetrize the flow (e.g., Figs. $3 \mathrm{~g}-\mathrm{i}$ where the $-\delta_{0} / f_{0} \times 10$ test is presented).

In all sensitivity tests except for the $\tau_{d}$ test, the NAMI decreases quasi linearly with time toward zero by $t^{\prime}=3$, but it never reaches zero due to the ceaseless convection that produces asymmetry and nonmonotonicity (Fig. 11). The $\tau_{d}$ case decreases much more slowly due to the damped vortex interaction. The $-\widetilde{\Delta h} / H \times 1 / 8$ test yields a much lower NAMI than the reference test, due to the more fine-grained, and therefore more homogeneous and axisymmetric forcing. Its standard deviation is the smallest among all, because the limit of a fine-grained mode is the full determinacy. The $\widetilde{r_{u}} / R \times 2$ test also yields a low NAMI. In this case, a wider updraft spins up a wider early-stage vortex, and the convective frequency is also smaller (for a fixed $\delta_{0}$ ). As a result, the length scale of forcing is larger, and the system requires fewer merger events to form a major vortex. This explains why its NAMI is already small near the beginning. If we interpret the $\widetilde{r_{u}} / R \times 2$ test as halving $R$, we judge that for a fixed $\delta_{0}$ (convective vigor), a smaller MCS leads to faster axisymmetrization of the vortex. Kilroy and Smith (2017) also reported faster axisymmetrization for a smaller initial midlevel vortex (roughly equivalent to our $R$ ) in their 3D cloud-resolving simulation. However, the controlled variable and therefore the perspective is different. Their initial midlevel vortex's vorticity is set to be larger for a smaller vortex, and they interpreted the faster axisymmetrization as the higher convective vigor caused by the higher boundary layer top pumping velocity. The NAMI is smaller for a higher viscosity $(\operatorname{Re} \times 1 / 2)$, due to the stronger damping on the filaments. The convective duration time $-\delta_{0} \widetilde{T_{u}}$ has little influence on NAMI for the parameters we study.

\section{c. Intensity}

What determines the maximum wind (intensity) of a vortex? First, the circulation theorem tells that a vortex with a higher vorticity magnitude (more compact) should have a larger maximum wind. A more compact vortex also has a higher inertial stability, as well as a higher survivability in a strain field (Dritschel 1990). A point vortex, whose vorticity is concentrated in an infinitesimal core, is the most compact and robust one with an infinite maximum wind. Second, a lower (a) ref

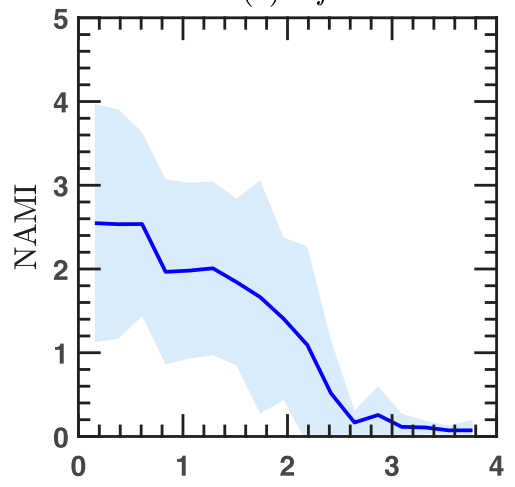

(d) $\widetilde{r_{u}} / R \times 2$

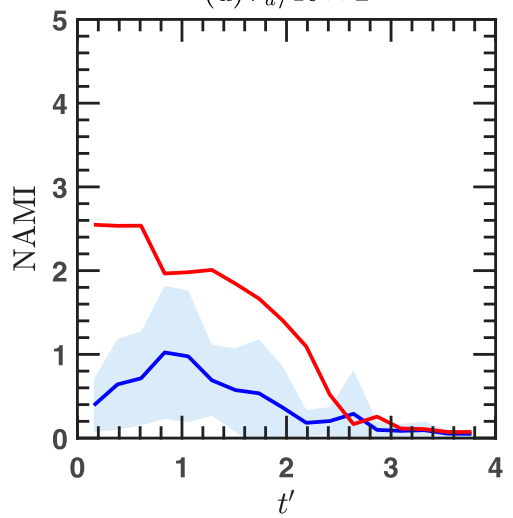

(b) $-\Delta h / H \times 1 / 8$

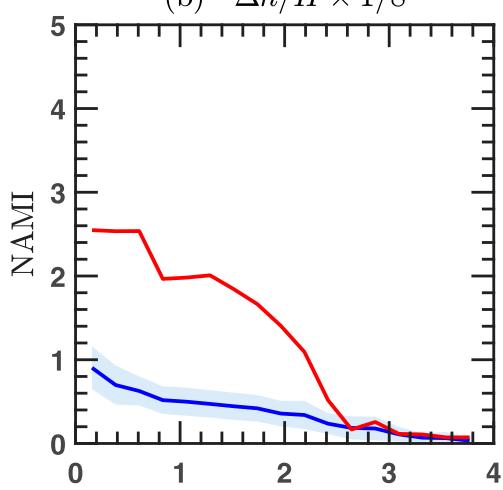

(e) $-\delta_{0} \widetilde{T_{u}} \times 1 / 4$

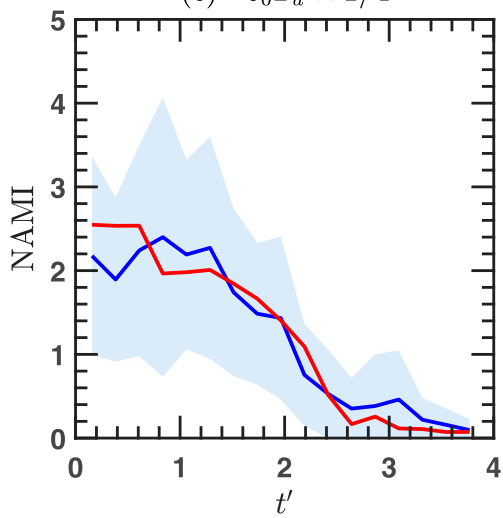

(c) $-\delta_{0} \tau_{d}=2$

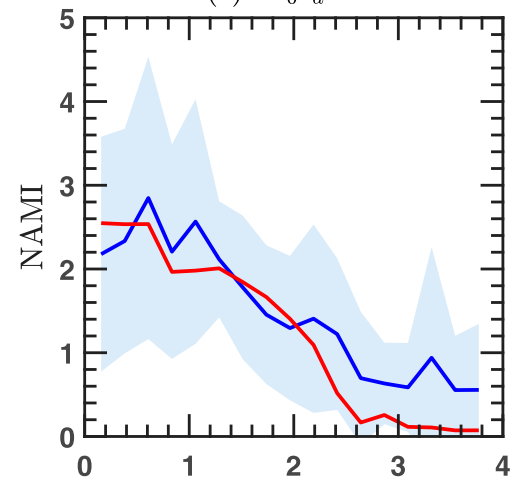

(f) $\operatorname{Re} \times 1 / 2$

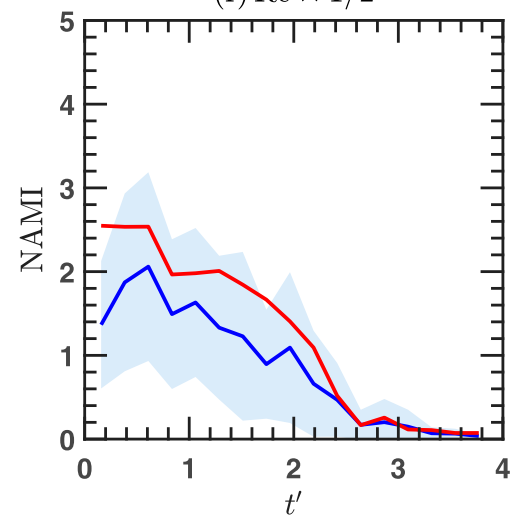

FIG. 11. The time evolution of NAMI (nonaxisymmetric and nonmonotonic index) with time for the six tests introduced in Fig. 9. The solid blue line is the ensemble average value in each test. The blue shadow denotes the \pm 1 standard deviation range. (b)-(f) The red line denotes the NAMI of the reference test, which is identical to the blue line in (a). 
NAMI enhances the maximum wind: an axisymmetric vorticity patch yields the highest peripheral velocity due to its shortest streamfunction contour. As a higher $-\Delta h / H$ leads to higher compactness and higher asymmetry at the same time, which factor dominates?

As the compactness is related to the convective intermittency, the least compact vortex is the uniform convergence case $(-\Delta h / H \rightarrow 0)$ discussed in section $5 \mathrm{~d}$. We now use the expression of $V_{b}$ and $r_{b}$ in (37) to understand the maximum azimuthal mean azimuthal wind $\overline{V_{m}}$ and its radius $r_{m}$ in the simulation. The $V_{b}$ rises with time and $r_{b}$ contracts with time. The $V_{b}$ consists of a linear growth regime at $-\delta_{0} t \lesssim 1$ where $V_{b} /\left(f_{0} R\right) \approx-\delta_{0} t / 2$, and an exponential growth regime at $-\delta_{0} t \gtrsim 1$ where $V_{b} \propto e^{-\delta_{0} t / 2}$. The growth is dominated by the stretching of a constant planetary vorticity in the first regime and the stretching of a growing relative vorticity in the second regime. The $V_{b}$ and $r_{b}$ provide a theoretical reference for the magnitude of $\overline{V_{m}}$ and $r_{m}$ for the finite $-\widetilde{\Delta h} / H$ cases.

Figure 12a shows that the maximum total wind $V_{\max }$ increases with $-\widetilde{\Delta h} / H$. Thus, the vorticity compactness dominates the asymmetry in determining the intensity. The $\widetilde{r_{u}} / R \times 2$ test has a larger $V_{\max }$ than the reference test at the early stage, in accordance with its lower NAMI at the early stage. In all tests, the initial jump of $V_{\max }$ for the finite $-\widetilde{\Delta h} / H$ tests is due to the convergent flow of updrafts. At the later stage where the convergent flow is far smaller than the rotational flow, all the $V_{\max }$ grows quasi exponentially at a rate of $-\delta_{0} / 2$, similar to $V_{b}$.

Figures $12 \mathrm{~b}$ and $12 \mathrm{c}$ show the dependence of $V_{\max }, \overline{V_{m}}$ and $r_{m}$ on $-\widetilde{\Delta h} / H$ at $t_{c}^{\prime}=2.98$ by which time the major vortex has roughly formed. The expectation and standard deviation of both $V_{\max }$ and $\overline{V_{m}}$ increase significantly with $-\widetilde{\Delta h} / H$. The expectation of $r_{m}$ drops with increasing $-\widetilde{\Delta h} / H$, featuring a more compact vortex, as is visualized in Fig. 10. While the lower bound of the expectation of $V_{\max }$ is provided by the $-\widetilde{\Delta h} / H \rightarrow 0$ case, the upper bound is provided by resorting the theoretical PDF for a given $-\widetilde{\Delta h} / H$ to the NAMI $\rightarrow 0$ state. The azimuthal velocity $V_{U}\left(r, t^{\prime}\right)$ of such a resorted vortex is transformed from the vorticity PDF using the differential form of (34):

$$
\begin{aligned}
\frac{V_{U}\left(r, t^{\prime}\right)}{f_{0} R}=\frac{1}{f_{0} R} & \frac{1}{r} \int_{0}^{r} \omega_{U}\left(r^{\prime}, t^{\prime}\right) r^{\prime} d r^{\prime} \\
& =\frac{R}{2 r} \int_{x^{\prime}}^{+\infty} \sigma\left(x^{\prime \prime}, t^{\prime}\right)\left(e^{x^{\prime \prime}}-1\right) d x^{\prime \prime}
\end{aligned}
$$

Here the resorted vorticity profile from the theoretical PDF is denoted as $\omega_{U}$. As the integral requires $\sigma$ value at the high $x^{\prime}$ range, we use the interpolated discrete PDF analytical solution (24), which is computationally more accurate and cheaper than the numerical solution of the continuous PDF Eq. (28). The maximum $V_{U}$ on the radial profile, $V_{U m} \equiv$ $\max \left\{V_{U}\right\}$, is the theoretical upper bound. It increases with $-\widetilde{\Delta h} / H$ (Fig. 12b). The corresponding radius $r_{U m}$, which is a lower bound of $r_{m}$, decreases with $-\widetilde{\Delta h} / H$ (Fig. 12c). They encapsulate most of the WTGE result. In another view, this reconstructed vortex is driven by an equivalent axisymmetric convergence which is resorted from the PDF of convergence (a pure Poisson distribution). A larger $-\widetilde{\Delta h} / H$ leads to a more compact convergence, and therefore a more compact vortex.

In a word, the model shows that more intermittent convection generally makes the major vortex more intense. The accompanying higher fluctuation on vorticity PDF and vorticity spatial distribution (higher NAMI) make the intensity less deterministic.
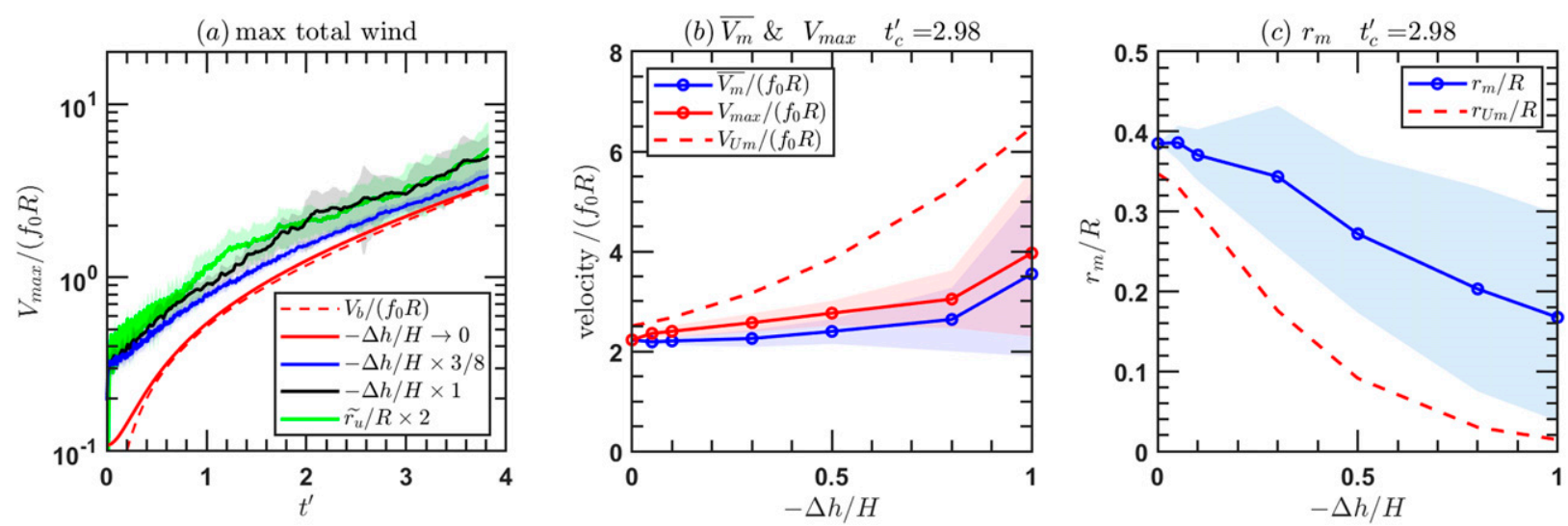

FIG. 12. (a) The solid red, blue, black, and green lines denote the ensemble average of the domain maximum wind, for the $-\Delta h / H \rightarrow 0$ test (the uniform convergence barotropic simulation with eddy diffusivity), the $-\Delta h / H \times 3 / 8$ test, the reference test, and the $\widetilde{r_{u}} / R \times 2$ test, respectively. The red dashed line denotes $V_{b}$, which is the velocity at the solid rotation vortex core boundary. (b) The ensemble average of the maximum azimuthal mean azimuthal wind $\overline{V_{m}}$ (blue circle line) and the maximum total wind $V_{\max }$ (red circle line) at $t_{c}^{\prime}=2.98$ for simulations with different $-\Delta h / H$. The dashed red line denotes the theoretical upper-bound $V_{U m}$. (c) The blue circle line is the ensemble average of $r_{m}$, which is the radius of $\overline{V_{m}}$, for different $-\Delta h / H$. The dashed red line denotes $r_{U m}$, which is the radius on the theoretically reconstructed profile $V_{U}$ where $V_{U m}$ resides. All changes on $-\Delta h / H$ are accompanied by the corresponding changes on $-\delta_{0} \widetilde{T_{u}}$ to keep the nondimensional mass sink rate $\widetilde{Q_{m}} /\left(-\delta_{0} H\right)$ fixed. All of the shadow denotes the \pm 1 standard deviation range of the 20 -member ensemble. 


\section{d. Pathway to parameterizing the eddy acceleration effect}

The wind profile $V_{U}$ obtained from the Lagrangian resorted vorticity PDF is not fully closed, because it only tells the growth rate with respect to $t^{\prime}=-\delta_{0} t$, rather than $t$. The next work is to parameterize the acceleration effect of the convectively generated eddies as a correction factor to the existing axisymmetric models that predict a time-varying $\delta_{0}$ (Raymond et al. 2007; Schönemann and Frisius 2012; Emanuel 2012). For our barotropic model, the governing equation of the azimuthal mean azimuthal wind $\overline{V_{\theta}}$ is

$$
\frac{\partial \overline{V_{\theta}}}{\partial t}=-\overline{V_{r}} \bar{\omega}-\overline{V_{r^{\prime}} \omega^{\prime}}-\frac{\overline{V_{\theta}}}{\tau_{d}}+\nu\left(\frac{\partial^{2} \overline{V_{\theta}}}{\partial r^{2}}+\frac{1}{r} \frac{\partial \overline{V_{\theta}}}{\partial r}-\frac{\overline{V_{\theta}}}{r^{2}}\right)
$$

with the overbar denoting azimuthal average. Here $\overline{V_{r}}$ is the azimuthal-averaged radial wind, and $V_{r}^{\prime}$ is its perturbation counterpart. The radial eddy vorticity flux $\overline{V_{r}^{\prime} \omega^{\prime}}$ is the eddy acceleration term.

In an Eulerian view, Nolan and Farrell (1999) solved $\overline{V_{r}^{\prime} \omega^{\prime}}$ as the linear response of an axisymmetric swirling vortex to asymmetric white-noise forcing. For a basic-state vortex without an eye (their "one-celled" case), they showed that only the high azimuthal wavenumber component of the forcing accelerates the flow. This solution is unsuitable for a tropical depression genesis problem whose major vortex is not much stronger than the asymmetric disturbance, and is highly unsteady (Nolan et al. 2007).

To avoid the two limitations, we propose a novel parameterization of $\overline{V_{r}^{\prime} \omega^{\prime}}$ by considering the inward (upgradient in the azimuthal-average sense) transport of vorticity as a linear relaxation of $\overline{V_{\theta}}$ to $V_{U}$ :

$$
\overline{V_{r}^{\prime} \omega^{\prime}}=-(\underbrace{\frac{V_{U}-\overline{V_{\theta}}}{\tau_{a}}}_{\text {upgrad }} \underbrace{+\nu_{a} \frac{\partial \bar{\omega}}{\partial r}}_{\text {downgrad }}) .
$$

The $\tau_{a}$ is the time scale of adjustment to axisymmetry, which can be taken to be the NAMI decaying time scale in Fig. 11. The $\nu_{a}$ is an eddy diffusivity that approximates the vorticity mixing in the adjustment process as a downgradient transport in the azimuthal-average sense. When $-\Delta h / H \rightarrow 0$, there should be $\nu_{a} \rightarrow 0$, so that $\overline{V_{r}^{\prime} \omega^{\prime}} \rightarrow 0$. We leave the careful evaluation of (41) and the quantification of $\tau_{a}$ and $\nu_{a}$ for future work.

\section{Comparison with a cloud-permitting simulation}

By analyzing the flow field, Kilroy et al. (2017) have found evidence of high-vorticity patch being produced by the accumulation of random stretching. To introduce the PDF view and validate that this process obeys a Markov chain, we run a cloud-permitting simulation of rotating radiative-convective equilibrium (RCE) problem (e.g., Bretherton et al. 2005) with the Bryan Cloud Model (CM1; Bryan and Fritsch 2002). The domain size is $1080^{2} \mathrm{~km}^{2}$, with doubly periodic boundary condition and a uniform sea surface temperature (SST). The grid interval is $2 \mathrm{~km}$, which roughly permits the existence of deep convection. The physical process is mostly identical to the full-physics "configured RCE test" in CM1. It uses Morrison double-moment microphysics scheme (Morrison et al. 2005), RRTMG radiation scheme, and the simple planetary boundary layer scheme by Bryan and Rotunno (2009). The only difference is the surface model where we choose "sfcmodel=3," the revised scheme for WRF Model. It provides a more realistic (higher) surface flux in RCE simulation than the default "sfcmodel=1."

This is a spontaneous tropical cyclogenesis problem without a prescribed initial vortex. This setup is clean and easy to implement, but the relevance to the real atmosphere which is full of disturbances and large-scale forcing is still in doubt (e.g., Dunkerton et al. 2009). As we focus on the vortex spinup from an existing MCS, whether the formation mechanism of the MCS is realistic is not a major concern. We set a relatively high SST $=305 \mathrm{~K}$, which is frequently used in spontaneous tropical cyclogenesis simulation (Wing et al. 2016; Carstens and Wing 2020). The Coriolis parameter is a constant value of $f_{0}=10^{-4} \mathrm{~s}^{-1}$. The motivation for using such a high $f_{0}$ is to make the generated tropical cyclone small enough to fit the domain (Khairoutdinov and Emanuel 2013; Muller and Romps 2018; Yang and Tan 2020). We refer the readers to Carstens and Wing (2020) and Ramsay et al. (2020) for a systematic sensitivity study on $f_{0}$ and SST, respectively. The initial sounding of potential temperature and vapor mixing ratio uses the horizontally averaged profile of a $120^{2}-\mathrm{km}^{2}$ small-domain nonrotating simulation with identical parameter setting run to the end of day 100 , by which time an equilibrium state has established. We have made several runs, with the initial noise (a maximum of $0.1 \mathrm{~K}$ on the boundary layer potential temperature) regenerated each time. In the chosen run, the multiple small convective clusters have merged into a single MCS before the low-level vortex significantly spins up. This is not the only path of tropical cyclogenesis, but is closer to the setup of our theory.

A convective cluster evolves out of the seemingly random convection by day 35 . It evolves to a $\sim 20 \mathrm{~m} \mathrm{~s}^{-1}$ tropical storm stage (American Meteorological Society 2012) by day 40, and then reaches a peak maximum surface wind around $80 \mathrm{~m} \mathrm{~s}^{-1}$ by day 43 , as is shown in Fig. 13a. Figures 14a-c show the $1.18-6.25-\mathrm{km}$ averaged vertical vorticity (regarded as the low-midlevel) by $t_{A}=35$ days, $t_{B}=37.5$ days, and $t_{C}=40$ days, which correspond roughly to the MCS formation time, a sample of vortex development time, and the peak time of the low-midlevel convergence. The last major merger event finishes by day 36.5 , marking the transition to an aggregated convective state with coarse-grained convection. Two reasons may contribute to the production of negative vorticity: the vorticity dipoles due to the tilting of horizontal vorticity (vertical shear) to the vertical by either updraft or downdraft (Kilroy et al. 2014), as well as the subsiding shell around the updraft which is driven by the evaporative cooling of hydrometeors or simply the nonhydrostatic compensating subsidence (Smith and Nicholls 2019). The vertical shear is part of the system circulation, which is omitted in our single-layer model. To further study the vorticity PDF, 
(a) $V_{\max }$ and CAPE

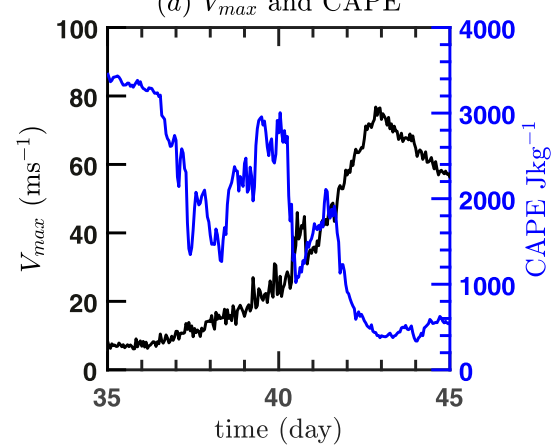

(b) central vort

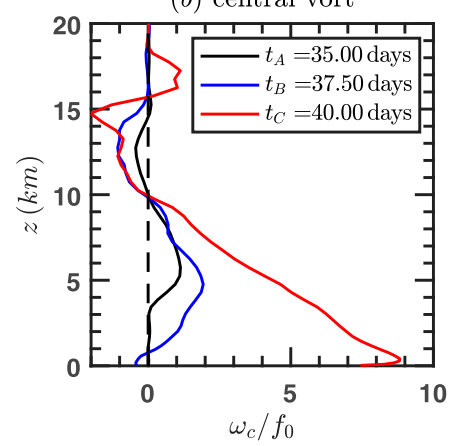

(c) $\delta_{0}$ and rainfall

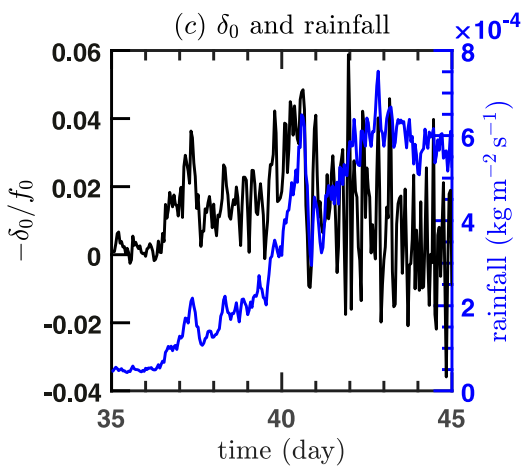

FIG. 13. (a) The black line is the maximum total wind at 25-m height (not at the low-midlevels) within the $R=250-\mathrm{km}$ MCS. The MCS center is defined as the maximum point of the filtered low-midlevel vertical vorticity, which is defined as the $1.18-6.25$-km vertically averaged vertical vorticity processed with a 30-km-scale horizontal Gaussian filter. The blue line is the time series of the 30-km-filtered convective available potential energy (CAPE) at the MCS center. (b) The vertical profile of the 30-km-filtered vertical relative vorticity at the MCS center $\omega_{c}$ normalized by $f_{0}$, with the black, blue, and red curve denoting $t_{A}=35$ days, $t_{B}=37.5$ days and $t_{C}=40$ days, respectively. (c) The black line is the time series of the MCS-averaged low-midlevel (1.18-6.25-km vertically averaged) nondimensional divergence $\delta_{0}(t) / f_{0}$, and the blue line is the MCS-averaged rainfall rate (unit: $\mathrm{kg} \mathrm{m}^{-2} \mathrm{~s}^{-1}$ ).

we need to define the MCS region and calculate the low-midlevel convergence.

We set the MCS size to be $R=250 \mathrm{~km}$ based on visual inspection of the vigorous convective region, as is indicated by the white circle in Fig. 14. The system (MCS) center is set as the maximum 30-km Gaussian filtered low-midlevel vertical vorticity. No density weighting is used in calculating the vertical average of vertical vorticity. The vertical structure of the filtered vertical vorticity at the vortex center is positive below $10 \mathrm{~km}$ and weakly negative between 10 and $15 \mathrm{~km}$ (Fig. $13 \mathrm{~b})$. At $t_{A}$, the vorticity is largest near $5-\mathrm{km}$ height, indicating the presence of a midlevel vortex. The maximum level decreases gradually toward the surface with time. This lowlevel spinup process, which is the focus of our theory, is frequently seen in observations (Houze et al. 2009; Bell and Montgomery 2019).

For the 3D simulation, $\delta_{0}$ is calculated as the $1.18-6.25-\mathrm{km}$ height averaged bulk divergence (without density weighting)
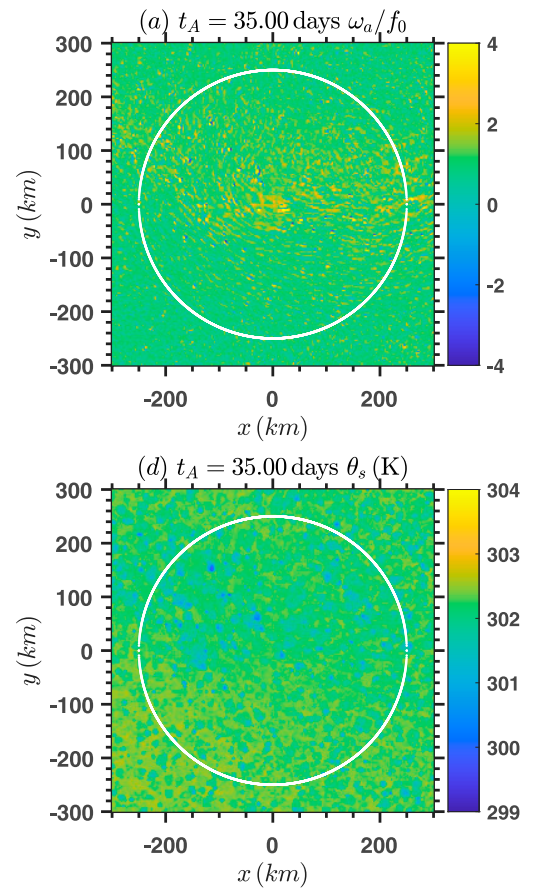
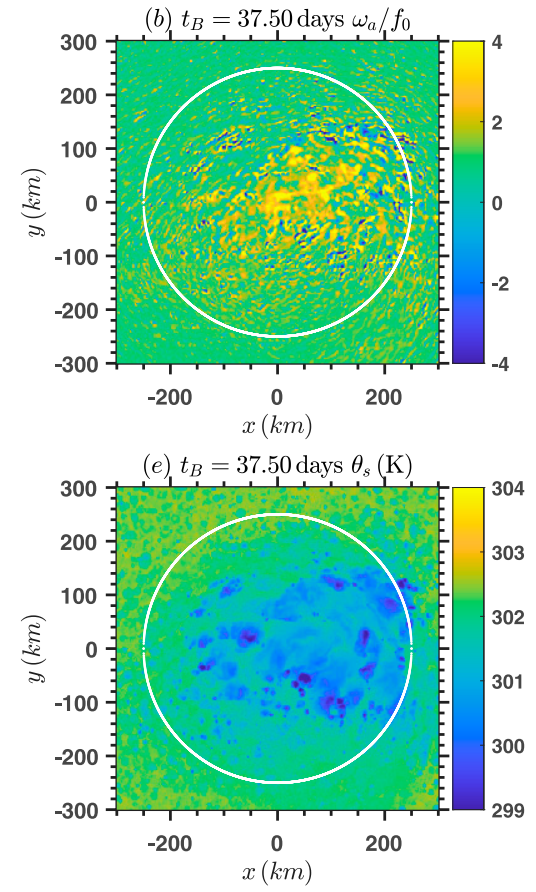
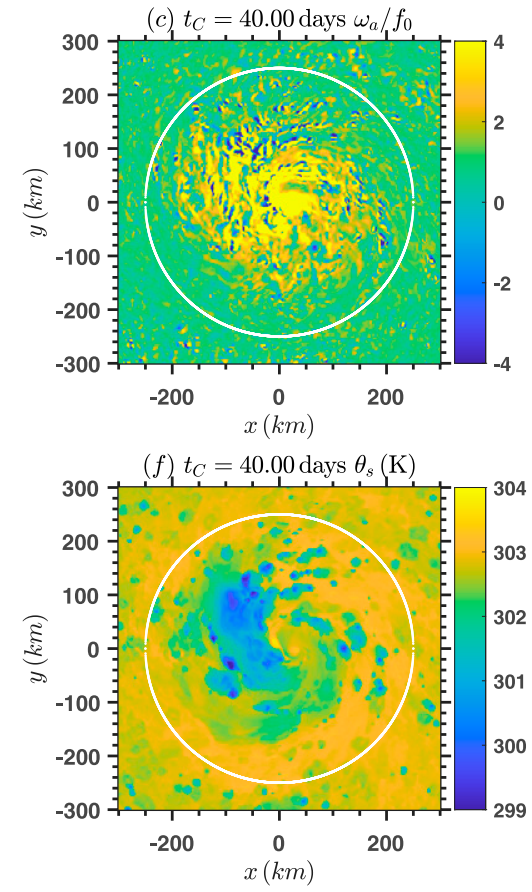

FIG. 14. (top) The low-midlevel (1.18-6.25-km vertically averaged) vertical absolute vorticity normalized by $f_{0}$ at (a) $t_{A}=35$ days, (b) $t_{B}=37.5$ days, and (c) $t_{C}=40$ days. (bottom ) The near surface $(z=25 \mathrm{~m})$ potential temperature $\theta_{s}$ at $(\mathrm{d}) t_{A}=35$ days, (e) $t_{B}=37.5$ days, and (f) $t_{C}=40$ days. 
within $R=250 \mathrm{~km}$ (Fig. 13c). It is only a coarse approximation to $\delta_{0}$, because it also contains the Ekman pumping-induced divergence. Its magnitude increases to around $0.04 f_{0}$ by day 40 and then decreases. There is an oscillation with a period of $\sim 0.5$ day, which we speculate to be due to either the stationary gravity wave trapped in the doubly periodic domain, or the coarse-grained convection in the MCS. The MCSaveraged rainfall rate, which represents the column net latent heat release, increases between days 35 and 43 (Fig. 13c). The different trend of the convergence and rainfall indicates the weakening of free-tropospheric entrainment by the growing inertial stability (Kilroy et al. 2017). This, together with the earlier drop of the central region convective available potential energy (CAPE; Fig. 13a) indicate that our one-layer model gradually becomes invalid after the maximum $-\delta_{0}$ time. The characteristic $-\delta_{0} / f_{0}$ in its climbing phase is around 0.02 . This is smaller than that used in our one-layer model which is suitable for the lower Coriolis parameter $\left(4.99 \times 10^{-5} \mathrm{~s}^{-1}\right)$.

The $\delta_{0}$ is used to rescale the time coordinate: $t^{\prime}=-\int_{t A}^{t} \delta_{0}\left(t^{\prime \prime}\right) d t^{\prime \prime}$, whose relation to the real time $t$ is shown in Fig. 15a. The initial $t^{\prime}=0$ time is chosen as $t_{A}$ where the MCS is just discernible. The $t_{B}$ and $t_{C}$ correspond to $t_{B}^{\prime}=0.19$ and $t_{C}^{\prime}=0.53$. Figure $15 \mathrm{~b}$ shows the system-averaged (within the radius $R$ ) low-midlevel vertical vorticity $\omega^{+}$roughly obeys $\omega^{+} / f_{0} \approx t^{\prime}$, in agreement with (14).

The low-midlevel vertical vorticity PDF at the three snapshots are shown in Fig. 16. On the positive vorticity side, the high-vorticity tail grows, and the slope flattens. This is qualitatively similar to the analytical solution of Markov chain in (24), which is calculated with $r_{u}=8 \sqrt{2} \mathrm{~km}$ (insensitive), $R=$ $250 \mathrm{~km}$ and $-\Delta h / H=2$. We have not figured out the method to diagnose or theoretically predict $-\Delta h / H$, so the $-\Delta h / H$ is subjectively chosen to roughly fit the PDF. However, we do notice that the cold pools become wider as the vortex develops (Figs. 14d-f), which may indicate the growth of $-\Delta h / H$ (Böing et al. 2012; Wang et al. 2019). After $t_{B}$, the cold pool gradually ceases due to the strong wind-induced surface heat flux. It is unclear what determines the convective intermittency at this later stage. In the future, the PDF model can be updated to include random tilting. Though the negative vorticity produced by the tilting occupies a smaller fractional area (e.g., Fig. 16b), the positive vorticity part of the dipole can be coupled to random stretching and produces air columns with high vorticity magnitude.

\section{Discussion}

This paper advances the understanding of the vorticity structure of a tropical depression, which determines its intensity and ability to survive in a straining environment. In an SWE model that mimics the lower troposphere, we put random mass sinks in a circular region to mimic convection in an MCS which is the precursor of tropical depression. The numerical simulation shows that the vorticity produced by repetitive convective stretching is aggregated to a large major vortex via both merger and the converging flow, qualitatively capturing the vorticity evolution reported by previous $3 \mathrm{D}$ simulations. As such a vortex has a quasi-monotonic vorticity radial distribution, its structure is linked to a vorticity PDF, which is the theme of this paper.

First, we show that the SWE satisfies the weak temperature gradient approximation in a typical tropical depression genesis problem where Froude number Fr is small. This makes convective heating equivalent to convergence, and potential vorticity equivalent to absolute vorticity $\omega_{a}$. Simulations show that the PDF depends mainly on random convective stretching and Rayleigh drag, and is modified by eddy mixing. When there is convective stretching alone, the PDF is approximately (a) $t^{\prime}$

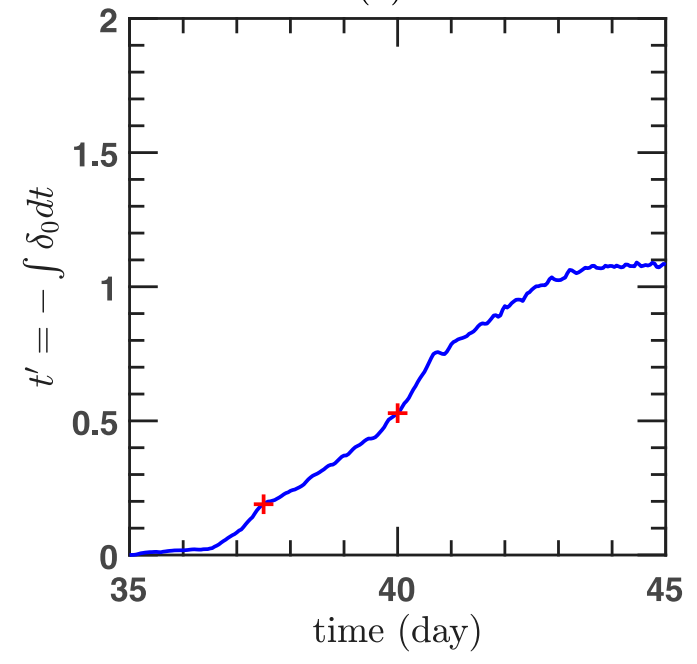

(b) MCS vort

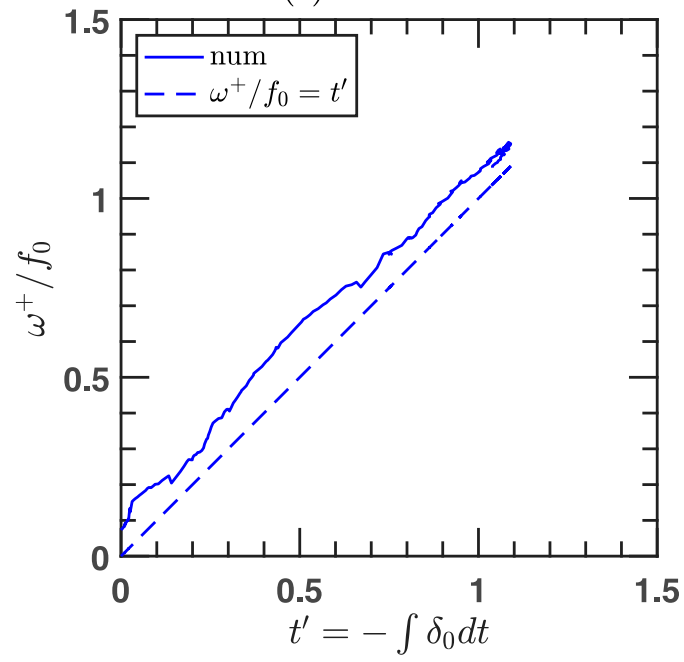

FIG. 15. (a) The blue curve is the rescaled nondimensional time coordinate $t^{\prime}=-\int_{t_{A}}^{t} \delta_{0}\left(t^{\prime \prime}\right) d t^{\prime \prime}$ vs time $t$. The red plus sign denotes $t_{B}^{\prime}$ and $t_{C}^{\prime}$. (b) The evolution of the MCS-averaged low-midlevel vertical vorticity $\omega^{+}$in $t^{\prime}$ coordinate. The solid blue line is from the simulation, and the dashed blue line denotes $\omega^{+} / f_{0}=t^{\prime}$. 
(a) positive vorticity PDF

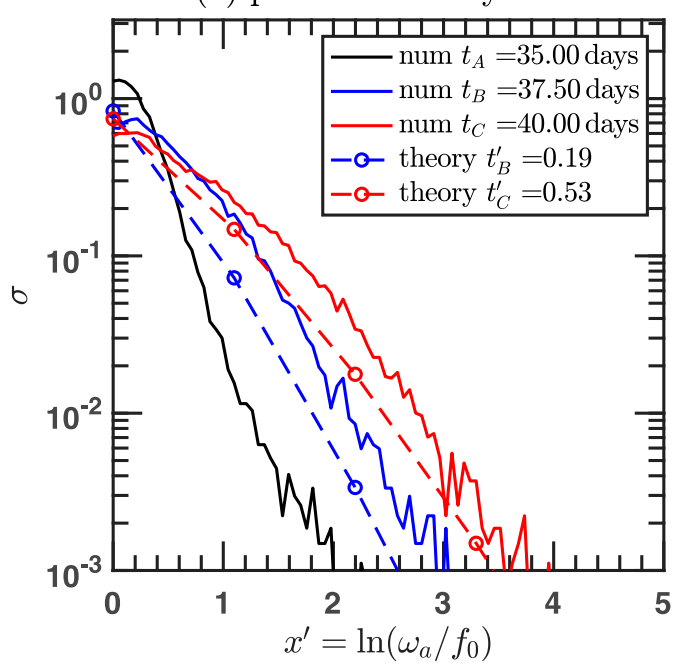

(b) negative vorticity PDF

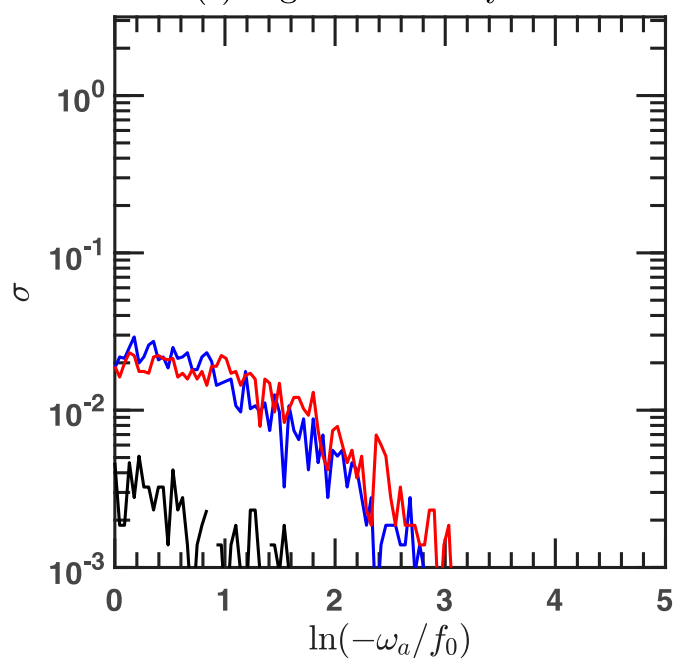

FIG. 16. The PDF of the low-midlevel (1.18-6.25-km vertically averaged) vertical absolute vorticity normalized by $f_{0}$. (a) The positive vorticity side $\left(\omega_{a}>f_{0}\right)$, with the solid black, blue, and red lines denoting $t_{A}=35$ days, $t_{B}=$ 37.5 days, and $t_{C}=40$ days, respectively. The rescaled nondimensional times are $t_{B}^{\prime}=-\int_{t_{A}}^{t_{B}} \delta_{0}\left(t^{\prime \prime}\right) d t^{\prime \prime}=0.19$ and $t_{C}^{\prime}=-\int_{t_{A}}^{t_{C}} \delta_{0}\left(t^{\prime \prime}\right) d t^{\prime \prime}=0.53$. The circled blue and red lines is the analytical solution of the discrete Markov chain shown in the first line of (24). (b) As in (a), but for the negative vorticity side $\left(\omega_{a}<-f_{0}\right)$. The Markov chain does not predict negative vorticity bins. The weak vorticity range $\left(-f_{0}<\omega_{a}<f_{0}\right)$ is not shown in this figure.

governed by a "Markov process" where air columns migrate on a set of discrete vorticity levels. Its analytical solution is a superposition of Poisson distributions weighted by the fraction of columns that enter the MCS at different times. Based on this, a better description that uses a hybrid PDF with the discrete base level $\omega_{a}=f_{0}$ and the continuous higher levels is established.

As has been verified by the one-layer model simulation, the PDF problem is governed by two nondimensional parameters: the accumulated convergence in a convective event $-\Delta h / H$ which measures the convective intermittency, and the Rayleigh drag scaled by MCS mean divergence $-\delta_{0} \tau_{d}$. The problem is self-similar to Coriolis parameter $f_{0}$ which nondimensionalizes $\omega_{a}$, and MCS mean divergence $\delta_{0}$ which nondimensionalizes $t$. For fixed $\delta_{0}$, a higher $-\Delta h / H$ represents a more coarse-grained convective mode that leads to a wider PDF with more high-vorticity columns, as well as a higher uncertainty (lower predictability). A shorter drag time scale $-\delta_{0} \tau_{d}$ damps vorticity magnitude and delays the vorticity aggregation.

An intense major vortex not only requires a wide PDF which provides high-vorticity columns to serve as a compact core, but also a more axisymmetric and monotonic vorticity spatial distribution. The newly introduced NAMI index (zero when fully axisymmetric and monotonic) drops significantly in a few system convergence time scales $\left(-\delta_{0}^{-1}\right)$. The system is more axisymmetric when convection is less intermittent, the $-\delta_{0} / f_{0}$ is smaller which enhances the eddy merger, and the $\widetilde{r_{u}} / R$ is larger which makes the scale separation between an updraft and the major vortex smaller. The intensity is quantified by the maximum total wind $V_{\max }$. Both the ensemble average and the standard deviation of $V_{\max }$ increase with $-\Delta h / H$. Thus, when convection is more intermittent, the expectation of vortex intensity is higher despite the stronger asymmetry, and its uncertainty is more significant. Note that the link of vorticity PDF with the monotonic vorticity structure and therefore wind distribution might be a privilege of the circular seeding (convective) geometry. If the seeding region is set as a band to simulate the intertropical convergence zone, the structure will suffer from shear instability, and a PDF model is not enough to tell the wind distribution.

The theory is compared to a cloud-permitting simulation of spontaneous tropical cyclogenesis with CM1 model, using the rescaled time coordinate $t^{\prime}=-\int \delta_{0} d t$. The PDF of 1.18 $6.25-\mathrm{km}$ height vertically averaged vertical vorticity within a 250-km convective region also shows the growth of the highvorticity tail, and the positive vorticity side qualitatively agrees with our Markov chain analytical solution.

Many more things could be done for investigating tropical cyclogenesis with this one-layer model:

- The turbulent process is only partly circumvented in the theory. It remains necessary to understand both the time scale of axisymmetrization $\left(\tau_{a}\right)$ and the eddy mixing effect on vorticity $\operatorname{PDF}\left(\nu_{a}\right)$. The latter is an important research topic in 2D nondivergent turbulence (e.g., Pasquero and Falkovich 2002).

- The convective scheme could be updated to consider the long-lived rotating convection which is identified in the simulation of Smith and Nicholls (2019). The interaction of convective vortices (Boubnov and Golitsyn 1986; Wang and Holland 1995; Schecter 2017), which is a deviation from a Markov process, should be considered.

- The model can be extended to consider more adverse factors that may prevent the MCS from developing into a hurricane. The survival ratio is important for tropical cyclone 
climatology (Hsieh et al. 2020). Apart from the drag, negative vorticity production by downdrafts should be considered. Perhaps the effect of horizontal shear and Rossby wave dispersion (on a beta plane) are most suitable to study, because they test the vortex's compactness, which we judge to depend on convective intermittency.

An extension to at least two vertical layers is needed to address the complicated vorticity pattern produced by tilting (Kilroy et al. 2014), as well as the transition from a stratiformdominated MCS with a midlevel vortex to a convectivedominated state with a low-level vortex (Montgomery et al. 2006).

Acknowledgments. The code for generating the figures, the code of the one-layer model, the namelist and initial sounding of the CM1 simulation, a derivation note, and a movie of the onelayer simulation are deposited in the online supplemental material. We are grateful to Prof. Sanjiva Lele and Prof. Nicholas Ouellette at Stanford University, Prof. Martin Singh at Monash
University, and Yunjiao Pu at Duke University for providing helpful comments. We thank Dr. Bolei Yang at Peking University for proofreading and suggestions. We thank Stanford University and Stanford Research Computing Center for providing computational resources and funding. We thank the two anonymous reviewers and Prof. Michael T. Montgomery for providing insightful comments. In the end, we wish our friend Prof. Adam Showman, who is far away, could receive our gratitude for his kind help.

\section{APPENDIX A}

\section{The Nondimensional SWE and WTGE Governing Equation}

Using $-\delta_{0}^{-1}$ as the time scale, $R$ as the horizontal length scale, $H$ as the vertical length scale, $-\delta_{0} R$ as the horizontal velocity scale, and $-\delta_{0} H$ as the vertical velocity scale, we introduce the nondimensional variables $t^{*}, \mathbf{x}^{*}, h^{*}, \omega^{*}, \delta^{*}$, $\mathbf{u}^{*}, Q_{u}^{*}, Q_{\mathrm{rad}}^{*}, \psi^{*}$ and $\phi^{*}$, which obey

$$
\begin{aligned}
& t=t^{*}\left(-\delta_{0}^{-1}\right), \quad \mathbf{x}=\mathbf{x}^{*} R, \quad h=h^{*} H, \quad \omega=\omega^{*}\left(-\delta_{0}\right), \quad \delta=\delta^{*}\left(-\delta_{0}\right), \quad \mathbf{u}=\mathbf{u}^{*}\left(-\delta_{0} R\right), \\
& Q_{u}=Q_{u}^{*}\left(-\delta_{0} H\right), \quad Q_{\mathrm{rad}}=Q_{\mathrm{rad}}^{*}\left(-\delta_{0} H\right), \quad \psi=\psi^{*}\left(-\delta_{0} R^{2}\right), \quad \phi=\phi^{*}\left(-\delta_{0} R^{2}\right) .
\end{aligned}
$$

The nondimensional gradient operator is $\nabla^{*} \equiv \mathbf{i} \partial / \partial x^{*}+\mathbf{j} \partial /$ $\partial y^{*}$. For SWE, we substitute (A1) into (1), (2), (8), and with the help of (10) to obtain

$$
\begin{gathered}
\frac{\partial h^{*}}{\partial t^{*}}+\nabla^{*} \cdot\left(\mathbf{u}^{*} h^{*}\right)=Q_{u}^{*}+Q_{\mathrm{rad}}^{*}+\frac{1}{\mathrm{Re}} \nabla^{* 2} h^{*}, \\
\frac{\partial \mathbf{u}^{*}}{\partial t^{*}}+\mathbf{u}^{*} \cdot \nabla^{*} \mathbf{u}^{*}+\left(\frac{-\delta_{0}}{f_{0}}\right)^{-1} \mathbf{k} \times \mathbf{u}^{*} \\
=-\mathrm{Bu}^{-1}\left(\frac{-\delta_{0}}{f_{0}}\right)^{-2} \nabla^{*} h^{*}+\frac{\mathbf{u}^{*}}{\delta_{0} \tau_{d}}+\frac{1}{\operatorname{Re}} \nabla^{* 2} \mathbf{u}^{*}, \\
Q_{u}^{*}+Q_{\mathrm{rad}}^{*}=\sum_{n=1}^{+\infty} Q_{n}^{*}\left(\mathbf{x}^{*}, t^{*}\right)+\frac{\pi R^{2}}{L^{2}}, \quad \text { with } \quad Q_{n}^{*}\left(\mathbf{x}^{*}, t^{*}\right) \\
=\widetilde{Q_{m}} e^{-\left|t^{*}+\delta_{0} t_{n}\right|^{2} /\left(\delta_{0} \widetilde{\tau}_{u}\right)^{2}-\left|\mathbf{x}^{*}-\mathbf{x}_{\mathrm{n}} / R\right|^{2} /\left(\widetilde{r_{u}} / R\right)^{2}}, \\
\widetilde{Q_{m}}{ }^{*}=\frac{\widetilde{Q_{m}}}{-\delta_{0} H}=-\frac{R^{2}}{\widetilde{r_{u}}} \frac{\Delta t}{\widetilde{T_{u}}}=-\frac{\widetilde{\Delta h}}{H} \frac{1}{\delta_{0} \widetilde{T_{u}}} .
\end{gathered}
$$

For WTGE, we substitute (A1) into (3), (6) and (7) to get

$$
\frac{\partial \omega^{*}}{\partial t^{*}}+\mathbf{u}^{*} \cdot \nabla^{*} \omega^{*}=-\delta^{*}\left(\omega^{*}-\frac{f_{0}}{\delta_{0}}\right)+\frac{\omega^{*}}{\delta_{0} \tau_{d}}+\frac{1}{\operatorname{Re}} \nabla^{* 2} \omega^{*},
$$

$$
\mathbf{u}^{*}=\mathbf{k} \times \nabla^{*} \psi^{*}+\nabla^{*} \phi^{*}, \quad \text { with } \quad \nabla^{* 2} \psi^{*}=\omega^{*}, \nabla^{* 2} \phi^{*}=\delta^{*},
$$

$$
\begin{gathered}
\delta^{*}\left(\mathbf{x}^{*}, t^{*}\right)=\sum_{n=1}^{+\infty} \delta_{u, n}^{*}\left(\mathbf{x}^{*}, t^{*}\right)+\frac{\pi R^{2}}{L^{2}}, \quad \text { with } \delta_{u, n}^{*}\left(\mathbf{x}^{*}, t^{*}\right) \\
=\delta_{u m}^{*} e^{-\left|t^{*}+\delta_{0} t_{n}\right|^{2} /\left(\delta_{0} \widetilde{\tau}_{u}\right)^{2}-\left|\mathbf{x}^{*}-\mathbf{x}_{n} / R\right|^{2} /\left(\widetilde{r_{u}} / R\right)^{2}}, \\
\delta_{u m}^{*}=\frac{\widetilde{Q_{m}}}{-\delta_{0} H}=-\frac{\widetilde{\Delta h}}{H} \frac{1}{\delta_{0} \widetilde{T}_{u}}
\end{gathered}
$$

The above equations show that there are six nondimensional parameters for WTGE: $-\delta_{0} / f_{0},-\widetilde{\Delta h} / H,-\delta_{0} \widetilde{T_{u}}$, $\widetilde{r_{u}} / R,-\delta_{0} \tau_{d}$, and Reynolds number $\operatorname{Re}=-\delta_{0} R^{2} / \nu$. For $\mathrm{SWE}$, there is an additional $\mathrm{Bu}=R^{2} / L_{R}^{2}=\left(f_{0} R\right)^{2} / c_{0}^{2}$, which accounts for the deviation from WTG.

\section{APPENDIX B}

\section{The Vorticity Production in a Single Updraft and the Subsequent Viscous Damping}

\section{a. The updraft stage}

Supposing the background is a (locally) solid body rotating flow with absolute vorticity $\omega_{a}=\omega_{a, 0}$. A convergence at a constant value of $-\delta_{u}$ is suddenly imposed to a circular 
region with a radius of $\widetilde{r_{u}}$ for $\widetilde{T_{u}}$ time. Such a top-hat convergence is a qualitative representation of the Gaussian profile used in the simulation. We assume that horizontal diffusion and drag are unimportant in such a short time. At $t=\widetilde{T_{u}}$, angular momentum conservation yields

$$
\omega_{a}= \begin{cases}\omega_{a, 0} e^{-\delta_{u} \widetilde{T_{u}}}, & \frac{r}{\widetilde{r_{u}}}<e^{\delta_{u}} \widetilde{T_{u} / 2}, \\ \omega_{a, 0}\left(\frac{r}{\widetilde{r_{u}}}\right)^{-2}, & \frac{r}{\widetilde{r_{u}}} \geq e^{\delta_{u}} \widetilde{T_{u} / 2}\end{cases}
$$

Note that a steady-state vorticity with a positive circular divergence forcing (balanced by Rayleigh drag) has been approximately solved by Sobel et al. (2001) to demonstrate their WTG framework. Air columns that are initially within $\widetilde{r_{u}}$ form a solid body rotation core. The outer region consists of columns entrained into the convection, so they are stretched less. The role of convection is concentrating vorticity, with the maximum absolute vorticity rising to $\omega_{a, 0} e^{-\delta_{u} T_{u}}$, and the length scale shrinking from $\widetilde{r_{u}}$ to $\widetilde{r_{u}} e^{\delta_{u} T_{u} / 2}$ where $\delta_{u} \widetilde{T_{u}}=\widetilde{\Delta h} / H$. The accumulated convergence $-\widetilde{\Delta h} / H$ can be understood as an area shrinking ratio.

\section{b. The postupdraft stage}

After the convection, the vorticity patch is susceptible to diffusion which will change its shape before the next convection hits it. The diffusion time scale $\tau_{\text {diff }}$ is estimated with $\nu$ and the vorticity patch length scale $l_{\mathrm{diff}}=\widetilde{r_{u}} e^{\delta_{u} T_{u} / 2}$. Now we apply these results to a Gaussian-shape convergence. Supposing the end state vorticity patch of a Gaussian updraft remains approximately Gaussian, we have $\nu \nabla^{2} \omega_{a} \sim 4 \nu \omega_{a} / l_{\text {diff }}^{2}$. Letting $\omega_{a} / \tau_{\text {diff }} \sim \nu \nabla^{2} \omega_{a}$, our theory predicts that the vorticity will be substantially diffused over $\tau_{\text {diff }}$ time:

$$
\tau_{\text {diff }} \sim \frac{l_{\text {diff }}^{2}}{4 \nu}=\frac{{\widetilde{r_{u}}}^{2}}{4 \nu} e^{\widetilde{\Delta h} / H} .
$$

We use a WTGE numerical simulation to demonstrate the damping by diffusion. A single updraft using the reference test value is put in the middle of a square domain with side length $L=120 \mathrm{~km}$ and a $256 \times 256$ mesh. The time step is $52.11 \mathrm{~s}$. Let $\omega_{a, 0}=f_{0}$. A Laplace viscosity of $\nu=160 \mathrm{~m}^{2} \mathrm{~s}^{-1}$ leads to $\tau_{\text {diff }}=2.01 \times 10^{4} \mathrm{~s}(0.23$ days $)$. Figure B1 shows that by $0.98 \tau_{\text {diff }}$ after the convective peak time, diffusion has smoothed the profile significantly.

Whether the patch will be significantly flattened by diffusion depends on a modified convective revisit time $\tau_{\mathrm{rev}, m}=$ $\left(-m \delta_{0}\right)^{-1}$ which is the convective interval for an air column to get involved in $m$ updrafts during one system convergence time scale $-\delta_{0}^{-1}$. The interval is shorter for luckier columns that receive multiple updrafts. Its ratio to $\tau_{\text {diff }}$ is

$$
\frac{\tau_{\mathrm{rev}, m}}{\tau_{\mathrm{diff}}}=\frac{4 \nu}{-\delta_{0}{\widetilde{r_{u}}}^{2}} \frac{e^{-\widetilde{\Delta h} / H}}{m}=4 \operatorname{Re}^{-1}\left(\frac{{\widetilde{r_{u}}}^{2}}{R^{2}}\right)^{-1} \frac{e^{-\widetilde{\Delta h} / H}}{m} .
$$

We get $\tau_{\text {rev }, m} / \tau_{\text {diff }} \approx 4.4 / m$ for the reference test, where $m$ is shown in Fig. 9 to be typically smaller than 5. Thus, diffusion could significantly flatten the shape before the next convection

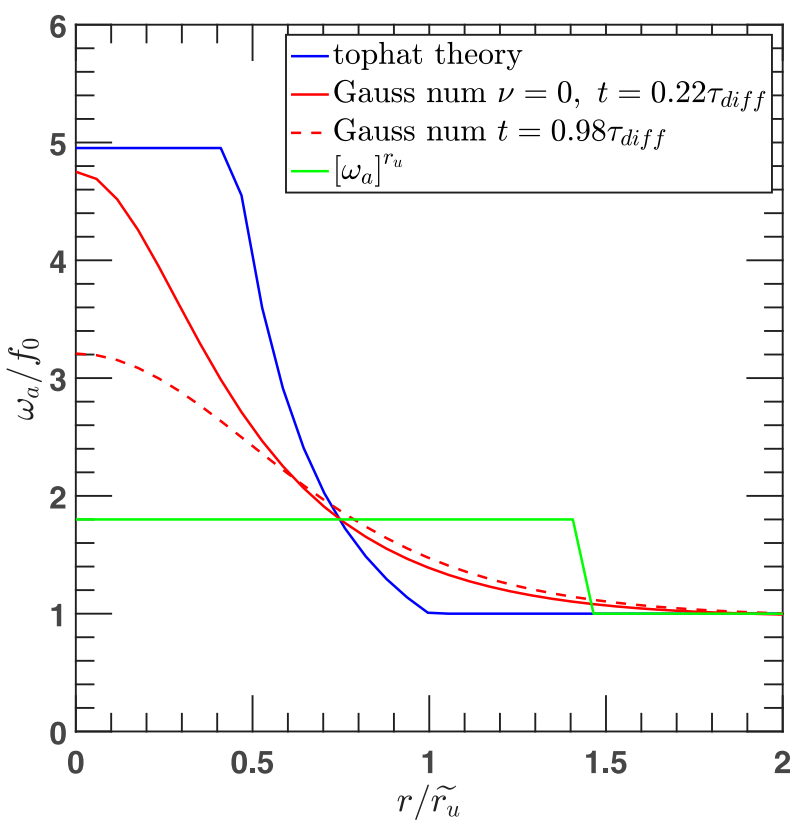

FIG. B1. The radial profile (the positive $x$ side of the $y=0$ cross section) of $\omega_{a} / f_{0}$ for the single updraft test. The peak time of updraft convergence is denoted as $t=0$. The solid blue line is the analytical solution, (B1), for the top-hat convergence profile, after the convection ends. The solid red line is an inviscid $(\nu=0)$ numerical solution of the Gaussian profile right after the convection ends. The dashed red line is a viscous $\left(\nu=160 \mathrm{~m}^{2} \mathrm{~s}^{-1}\right)$ simulation at $0.98 \tau_{\text {diff }}$ after the updraft peaks. The solid green line denotes the "vorticity-equivalent top-hat" profile of the vorticity patch which is used for the PDF model.

occurs. The ratio is larger for lower Re and higher $-\widetilde{\Delta h} / H$, but in their sensitivity tests in section 6a, a fixed $\alpha_{r}=\sqrt{2}$ works well in the vorticity PDF model. We explain such insensitivity as an automatic damping mechanism. Suppose multiple updrafts happen to fully or partially fall on the same vortical patch within a short time: the patch size will be several times smaller than $l_{\text {diff, }}$ so diffusion may serve as a "peak limiter" that preferentially damps the "lucky" high-vorticity patches, and the bulky dissipative effect may depend little on Re.

\section{APPENDIX C}

\section{A Finite-Domain Model of the MCS-Average Vorticity $\omega^{+}$with Rayleigh Drag}

We extend the model of $\omega^{+}$from an infinite domain to a finite domain, and add Rayleigh drag. The motivation is to account for the situation where multiple MCSs are not that far from each other, and for the artificial doubly periodic domain effect in simulations. A fixed $\delta_{0}$ and Rayleigh drag are used. We solve both $\omega^{+}$and the mean relative vorticity outside of the MCS: $\omega^{-}$. We ask, How large a domain is needed to neglect the influence of compensating divergence on $\omega^{+}$?

First, we introduce the MCS region net convergence $\left(-\delta_{0}^{+}\right)$that considers radiative cooling within it: 


$$
\delta_{0}^{+}=\delta_{0}\left(1-\frac{S^{+}}{S^{+}+S^{-}}\right)
$$

where $S^{+}=\pi R^{2}$ and $S^{-}=L^{2}-S^{+}$is the area of the MCS and that outside of the MCS, respectively. Performing area integration on the relative vorticity equation, Eq. (6), (in flux form) within the MCS region, we get

$$
\begin{aligned}
\frac{d \omega^{+}}{d t} & =-\frac{1}{\pi R^{2}} \oint_{\mathrm{MCS}} V_{r} \omega d l-f_{0} \delta_{0}^{+}-\frac{\omega^{+}}{\tau_{d}} \\
& \approx-\frac{2}{R} V_{r b} \omega^{-}-f_{0} \delta_{0}^{+}-\frac{\omega^{+}}{\tau_{d}} .
\end{aligned}
$$

Here $V_{r b}$ is the mean radial inflow velocity at the MCS boundary obtained from Gauss theorem. Note that the formulation of (C2) strictly only works under WTG. For SWE, the system-averaged divergence does not exactly equal to $\delta_{0}^{+}$due to the buildup of layer thickness anomaly (warm core). We have assumed the inflow vorticity value is equal to $\omega^{-}$, which is related to $\omega^{+}$by enforcing zero total relative vorticity of the doubly periodic domain:

$$
S^{-} \omega^{-}+S^{+} \omega^{+}=0 \text { and } \quad V_{r b}=\frac{\delta_{0}^{+} R}{2} .
$$

Thus, a larger domain relative to the MCS region leads to a more dilute descent, a smaller magnitude of $\omega^{-}$, and a more negligible inflow vorticity flux. The horizontal eddy mixing inside the MCS only redistributes vorticity there, so it does not influence $\omega^{+}$and $\omega^{-}$. Substituting (C3) into (C2), we obtain an ordinary differential equation for $\omega^{+}$:

$$
\frac{d \omega^{+}}{d t}=-\frac{\omega^{+}}{\tau_{d}^{+}}-f_{0} \delta_{0}^{+}, \quad \text { with } \tau_{d}^{+}=\left(-\delta_{0}^{+} \frac{S^{+}}{S^{-}}+\frac{1}{\tau_{d}}\right)^{-1} .
$$

Here $\tau_{d}^{+}$is an effective damping time scale of $\omega^{+}$. With zero relative vorticity as the initial condition, the solution is

$$
\frac{\omega^{+}}{f_{0}}=-\delta_{0}^{+} \tau_{d}^{+}\left(1-e^{-t / \tau_{d}^{+}}\right)
$$

When the MCS only takes a small fraction of the domain and the Rayleigh drag is not considered, $\tau_{d}^{+}$is much longer than the system development time scale $-\delta_{0}^{-1}$. In this case, the lowest-order approximation is (14): $\omega^{+} / f_{0} \approx-\delta_{0} t$, and the more exact solution in (C5) has a small curvature due to the local descent and the inflow of negative vorticity into the MCS. Figure 2a shows that the $\omega^{+}$of the WTGE for both the reference and the drag sensitivity test $\left(-\delta_{0} \tau_{d}=2\right)$ are in good agreement with (C5). There is no discernible difference between SWE and WTGE on the reference test, which validates the WTG approximation on the system scale. In all cases, we have $\left|\omega^{-}\right| \ll f_{0}$ for both SWE and WTGE. Thus, our $R / L=1 / 8$ setup is large enough for the negative vorticity transported into the MCS to be close to 0 , a property used by the vorticity PDF model in section 5 .

\section{REFERENCES}

American Meteorological Society, 2012: Glossary of Meteorology. Amer. Meteor. Soc., http://glossary.ametsoc.org.

Bell, M. M., and M. T. Montgomery, 2019: Mesoscale processes during the genesis of Hurricane Karl (2010). J. Atmos. Sci., 76, 2235-2255, https://doi.org/10.1175/JAS-D-18-0161.1.

Bister, M., and K. A. Emanuel, 1997: The genesis of Hurricane Guillermo: TEXMEX analyses and a modeling study. Mon. Wea. Rev., 125, 2662-2682, https://doi.org/10.1175/15200493(1997) $125<2662$ :TGOHGT>2.0.CO;2.

Böing, S. J., H. J. Jonker, A. P. Siebesma, and W. W. Grabowski, 2012: Influence of the subcloud layer on the development of a deep convective ensemble. J. Atmos. Sci., 69, 2682-2698, https://doi.org/10.1175/JAS-D-11-0317.1.

Boubnov, B., and G. Golitsyn, 1986: Experimental study of convective structures in rotating fluids. J. Fluid Mech., 167, 503531, https://doi.org/10.1017/S002211208600294X.

Bretherton, C. S., P. N. Blossey, and M. Khairoutdinov, 2005: An energy-balance analysis of deep convective self-aggregation above uniform SST. J. Atmos. Sci., 62, 4273-4292, https://doi. org/10.1175/JAS3614.1.

Bryan, G. H., and J. M. Fritsch, 2002: A benchmark simulation for moist nonhydrostatic numerical models. Mon. Wea. Rev., 130, 2917-2928, https://doi.org/10.1175/15200493(2002)130<2917:ABSFMN>2.0.CO;2.

- , and R. Rotunno, 2009: The maximum intensity of tropical cyclones in axisymmetric numerical model simulations. Mon. Wea. Rev., 137, 1770-1789, https://doi.org/10.1175/2008MWR2709.1.

Carstens, J. D., and A. A. Wing, 2020: Tropical cyclogenesis from self-aggregated convection in numerical simulations of rotating radiative-convective equilibrium. J. Adv. Model. Earth Syst., 12, e2019MS002020, https://doi.org/10.1029/2019MS002020.

Charney, J. G., and A. Eliassen, 1964: On the growth of the hurricane depression. J. Atmos. Sci., 21, 68-75, https://doi.org/10. 1175/1520-0469(1964)021<0068:OTGOTH > 2.0.CO;2.

Davis, C. A., 2015: The formation of moist vortices and tropical cyclones in idealized simulations. J. Atmos. Sci., 72, 3499 3516, https://doi.org/10.1175/JAS-D-15-0027.1.

Dritschel, D. G., 1990: The stability of elliptical vortices in an external straining flow. J. Fluid Mech., 210, 223-261, https:// doi.org/10.1017/S0022112090001276.

— , and D. Waugh, 1992: Quantification of the inelastic interaction of unequal vortices in two-dimensional vortex dynamics. Phys. Fluids, 4, 1737-1744, https://doi.org/10.1063/1.858394.

Dunkerton, T. J., M. T. Montgomery, and Z. Wang, 2009: Tropical cyclogenesis in a tropical wave critical layer: Easterly waves. Atmos. Chem. Phys., 9, 5587-5646, https://doi.org/10. 5194/acp-9-5587-2009.

Durran, D. R., 2010: Numerical Methods for Fluid Dynamics: With Applications to Geophysics. Vol. 32. Springer Science and Business Media, 533 pp.

Emanuel, K. A., 1986: An air-sea interaction theory for tropical cyclones. Part I: Steady-state maintenance. J. Atmos. Sci., 43, 585-605, https://doi.org/10.1175/1520-0469(1986)043<0585: AASITF $>2.0 . \mathrm{CO} ; 2$.

_ 1989: The finite-amplitude nature of tropical cyclogenesis. J. Atmos. Sci., 46, 3431-3456, https://doi.org/10.1175/15200469(1989)046<3431:TFANOT >2.0.CO;2.

, 1994: Atmospheric Convection. Oxford University Press, $580 \mathrm{pp}$. 
, 1997: Some aspects of hurricane inner-core dynamics and energetics. J. Atmos. Sci., 54, 1014-1026, https://doi.org/10. 1175/1520-0469(1997)054<1014:SAOHIC >2.0.CO;2.

_ 2012: Self-stratification of tropical cyclone outflow. Part II: Implications for storm intensification. J. Atmos. Sci., 69, 988-996, https://doi.org/10.1175/JAS-D-11-0177.1.

Enagonio, J., and M. T. Montgomery, 2001: Tropical cyclogenesis via convectively forced vortex Rossby waves in a shallow water primitive equation model. J. Atmos. Sci., 58, 685-706, https:// doi.org/10.1175/1520-0469(2001)058<0685:TCVCFV>2.0.CO;2.

Fang, J., and F. Zhang, 2011: Evolution of multiscale vortices in the development of Hurricane Dolly (2008). J. Atmos. Sci., 68, 103-122, https://doi.org/10.1175/2010JAS3522.1.

Ferreira, R. N., W. H. Schubert, and J. J. Hack, 1996: Dynamical aspects of twin tropical cyclones associated with the MaddenJulian oscillation. J. Atmos. Sci., 53, 929-945, https://doi.org/ 10.1175/1520-0469(1996)053<0929:DAOTTC > 2.0.CO;2.

Fritsch, J., J. Murphy, and J. Kain, 1994: Warm core vortex amplification over land. J. Atmos. Sci., 51, 1780-1807, https://doi. org/10.1175/1520-0469(1994)051<1780:WCVAOL>2.0.CO;2.

Gray, W. M., 1998: The formation of tropical cyclones. Meteor. Atmos. Phys., 67, 37-69, https://doi.org/10.1007/BF01277501.

Haerter, J. O., 2019: Convective self-aggregation as a cold pooldriven critical phenomenon. Geophys. Res. Lett., 46, 40174028, https://doi.org/10.1029/2018GL081817.

Hendricks, E. A., and W. Schubert, 2009: Transport and mixing in idealized barotropic hurricane-like vortices. Quart. J. Roy. Meteor. Soc., 135, 1456-1470, https://doi.org/10.1002/qj.467.

— M. T. Montgomery, and C. A. Davis, 2004: The role of "vertical" hot towers in the formation of Tropical Cyclone Diana (1984). J. Atmos. Sci., 61, 1209-1232, https://doi.org/10. 1175/1520-0469(2004)061<1209:TROVHT>2.0.CO;2.

- W. W. Schubert, Y.-H. Chen, H.-C. Kuo, and M. S. Peng, 2014: Hurricane eyewall evolution in a forced shallow-water model. J. Atmos. Sci., 71, 1623-1643, https://doi.org/10.1175/ JAS-D-13-0303.1.

Holloway, C. E., A. A. Wing, S. Bony, C. Muller, H. Masunaga, T. S. L'Ecuyer, D. D. Turner, and P. Zuidema, 2017: Observing convective aggregation. Surv. Geophys., 38, 1199-1236, https://doi.org/10.1007/s10712-017-9419-1.

Houze, R. A., Jr., W.-C. Lee, and M. M. Bell, 2009: Convective contribution to the genesis of Hurricane Ophelia (2005). Mon. Wea. Rev., 137, 2778-2800, https://doi.org/10.1175/ 2009MWR2727.1.

Hsieh, T.-L., G. A. Vecchi, W. Yang, I. M. Held, and S. T. Garner, 2020: Large-scale control on the frequency of tropical cyclones and seeds: A consistent relationship across a hierarchy of global atmospheric models. Climate Dyn., 55, 31773196, https://doi.org/10.1007/s00382-020-05446-5.

Julien, K., S. Legg, J. McWilliams, and J. Werne, 1999: Plumes in rotating convection. Part 1. Ensemble statistics and dynamical balances. J. Fluid Mech., 391, 151-187, https://doi.org/10.1017/ S0022112099005236.

Khairoutdinov, M., and K. Emanuel, 2013: Rotating radiative-convective equilibrium simulated by a cloud-resolving model. $J$. Adv. Model. Earth Syst., 5, 816-825, https://doi.org/10.1002/ 2013MS000253.

Kilroy, G., and R. K. Smith, 2017: The effects of initial vortex size on tropical cyclogenesis and intensification. Quart. J. Roy. Meteor. Soc., 143, 2832-2845, https://doi.org/10.1002/qj.3134.

_ - — , and U. Wissmeier, 2014: Tropical convection: The effects of ambient vertical and horizontal vorticity. Quart. J. Roy. Meteor. Soc., 140, 1756-1770, https://doi.org/10.1002/qj.2261.
,-- , and M. T. Montgomery, 2017: A unified view of tropical cyclogenesis and intensification. Quart. J. Roy. Meteor. Soc., 143, 450-462, https://doi.org/10.1002/qj.2934.

Lahaye, N., and V. Zeitlin, 2016: Understanding instabilities of tropical cyclones and their evolution with a moist convective rotating shallow-water model. J. Atmos. Sci., 73, 505-523, https://doi.org/10.1175/JAS-D-15-0115.1.

McWilliams, J. C., 1990: The vortices of two-dimensional turbulence. J. Fluid Mech., 219, 361-385, https://doi.org/10.1017/ S0022112090002981.

Middlebrooke, M. G., 1988: Investigation of tropical cyclone genesis and development using low-level aircraft flight data. Colorado State University Dept. of Atmospheric Science Paper 429, $94 \mathrm{pp}$.

Montgomery, M. T., and R. J. Kallenbach, 1997: A theory for vortex Rossby-waves and its application to spiral bands and intensity changes in hurricanes. Quart. J. Roy. Meteor. Soc., 123, 435-465, https://doi.org/10.1002/qj.49712353810.

— sification. Aust. Meteor. Oceanogr. J., 64, 37-66, https://doi. org/10.22499/2.6401.005.

- , and — 2017: Recent developments in the fluid dynamics of tropical cyclones. Annu. Rev. Fluid Mech., 49, 541-574, https://doi.org/10.1146/annurev-fluid-010816-060022.

— , H. D. Snell, and Z. Yang, 2001: Axisymmetric spindown dynamics of hurricane-like vortices. J. Atmos. Sci, 58, 421-435, https://doi.org/10.1175/1520-0469(2001)058<0421:ASDOHL >2.0. $\mathrm{CO} ; 2$.

- M. Nicholls, T. Cram, and A. Saunders, 2006: A vortical hot tower route to tropical cyclogenesis. J. Atmos. Sci., 63, 355-386, https://doi.org/10.1175/JAS3604.1.

Morrison, H., J. Curry, and V. Khvorostyanov, 2005: A new double-moment microphysics parameterization for application in cloud and climate models. Part I: Description. J. Atmos. Sci., 62, 1665-1677, https://doi.org/10.1175/JAS3446.1.

Muller, C. J., and D. M. Romps, 2018: Acceleration of tropical cyclogenesis by self-aggregation feedbacks. Proc. Natl. Acad. Sci. USA, 115, 2930-2935, https://doi.org/10.1073/pnas. 1719967115.

Neelin, J. D., and I. M. Held, 1987: Modeling tropical convergence based on the moist static energy budget. Mon. Wea. Rev., 115, 3-12, https://doi.org/10.1175/1520-0493(1987)115<0003: MTCBOT>2.0.CO;2.

Nolan, D. S., and B. F. Farrell, 1999: The intensification of twodimensional swirling flows by stochastic asymmetric forcing. J. Atmos. Sci., 56, 3937-3962, https://doi.org/10.1175/15200469(1999)056<3937:TIOTDS>2.0.CO;2.

tion from asymmetric convection: Energetics and efficiency. $J$. Atmos. Sci., 64, 3377-3405, https://doi.org/10.1175/JAS3988.1.

O’Neill, M. E., K. A. Emanuel, and G. R. Flierl, 2015: Polar vortex formation in giant-planet atmospheres due to moist convection. Nat. Geosci., 8, 523-526, https://doi.org/10.1038/ ngeo2459.

,-- , and -2016 : Weak jets and strong cyclones: Shallow-water modeling of giant planet polar caps. J. Atmos. Sci., 73, 1841-1855, https://doi.org/10.1175/JAS-D-15-0314.1.

Ooyama, K., 1969: Numerical simulation of the life cycle of tropical cyclones. J. Atmos. Sci., 26, 3-40, https://doi.org/10.1175/ 1520-0469(1969)026<0003:NSOTLC >2.0.CO;2.

Pasquero, C., and G. Falkovich, 2002: Stationary spectrum of vorticity cascade in two-dimensional turbulence. Phys. Rev. E, 65, 056305, https://doi.org/10.1103/PhysRevE.65.056305. 
Peters, J. M., C. J. Nowotarski, and G. L. Mullendore, 2020: Are supercells resistant to entrainment because of their rotation? J. Atmos. Sci., 77, 1475-1495, https://doi.org/10.1175/JAS-D-19-0316.1.

Pishro-Nik, H., 2014: Introduction to Probability, Statistics, and Random Processes. Kappa Research, LLC, 732 pp.

Pope, S. B., 2001: Turbulent Flows. IOP Publishing, 771 pp.

Ramsay, H. A., M. S. Singh, and D. R. Chavas, 2020: Response of tropical cyclone formation and intensification rates to climate warming in idealized simulations. J. Adv. Model. Earth Syst., 12, e2020MS002086, https://doi.org/10.1029/2020MS002086.

Raymond, D. J., S. L. Sessions, and Ž. Fuchs, 2007: A theory for the spinup of tropical depressions. Quart. J. Roy. Meteor. Soc., 133, 1743-1754, https://doi.org/10.1002/qj.125.

Ritchie, E. A., and G. J. Holland, 1997: Scale interactions during the formation of Typhoon Irving. Mon. Wea. Rev., 125, 1377-1396, https:// doi.org/10.1175/1520-0493(1997)125<1377:SIDTFO>2.0.CO;2.

_ and - 1999: Large-scale patterns associated with tropical cyclogenesis in the western Pacific. Mon. Wea. Rev., 127, 2027-2043, https://doi.org/10.1175/1520-0493(1999)127<2027: LSPAWT $>2.0 . \mathrm{CO} ; 2$.

Rotunno, R., and K. A. Emanuel, 1987: An air-sea interaction theory for tropical cyclones. Part II: Evolutionary study using a nonhydrostatic axisymmetric numerical model. J. Atmos. Sci, 44, 542-561, https:// doi.org/10.1175/1520-0469(1987)044<0542:AAITFT>2.0.CO;2.

Schecter, D. A., 2017: A computational study on the nature of meso- $\beta$ scale vortex coalescence in a tropical atmosphere. $J$. Adv. Model. Earth Syst., 9, 1366-1398, https://doi.org/10.1002/ 2016 MS000777.

— , and T. J. Dunkerton, 2009: Hurricane formation in diabatic Ekman turbulence. Quart. J. Roy. Meteor. Soc., 135, 823-838, https://doi.org/10.1002/qj.405.

Schönemann, D., and T. Frisius, 2012: Dynamical system analysis of a low-order tropical cyclone model. Tellus, 64A, 15817, https://doi.org/10.3402/tellusa.v64i0.15817.

Schubert, W. H., M. T. Montgomery, R. K. Taft, T. A. Guinn, S. R. Fulton, J. P. Kossin, and J. P. Edwards, 1999: Polygonal eyewalls, asymmetric eye contraction, and potential vorticity mixing in hurricanes. J. Atmos. Sci., 56, 1197-1223, https://doi.org/10.1175/ 1520-0469(1999)056<1197:PEAECA > 2.0.CO;2.

Showman, A. P., 2007: Numerical simulations of forced shallowwater turbulence: Effects of moist convection on the largescale circulation of Jupiter and Saturn. J. Atmos. Sci., 64, 3132-3157, https://doi.org/10.1175/JAS4007.1.

Smith, R. K., 2000: The role of cumulus convection in hurricanes and its representation in hurricane models. Rev. Geophys., 38, 465-489, https://doi.org/10.1029/1999RG000080.

Smith, W. P., and M. E. Nicholls, 2019: On the creation and evolution of small-scale low-level vorticity anomalies during tropical cyclogenesis. J. Atmos. Sci., 76, 2335-2355, https://doi.org/ 10.1175/JAS-D-18-0104.1.

Sobel, A. H., J. Nilsson, and L. M. Polvani, 2001: The weak temperature gradient approximation and balanced tropical moisture waves. J. Atmos. Sci., 58, 3650-3665, https://doi.org/10. 1175/1520-0469(2001)058<3650:TWTGAA > 2.0.CO;2.

Sukhatme, J., and W. R. Young, 2011: The advection-condensation model and water-vapour probability density functions. Quart. J. Roy. Meteor. Soc., 137, 1561-1572, https://doi.org/10.1002/qj.869.

Tennekes, H., and J. L. Lumley, 2018: A First Course in Turbulence. MIT Press, 300 pp.
Terwey, W. D., and M. T. Montgomery, 2008: Secondary eyewall formation in two idealized, full-physics modeled hurricanes. J. Geophys. Res., 113, D12112, https://doi.org/10.1029/2007JD008897.

Thom, H. C. S., 1957: The frequency of hail occurrence. Arch. Meteor. Geophys. Bioklimatol., 8B, 185-194, https://doi.org/ 10.1007/BF02243687.

Thomson, S. I., and M. E. McIntyre, 2016: Jupiter's unearthly jets: A new turbulent model exhibiting statistical steadiness without large-scale dissipation. J. Atmos. Sci., 73, 1119-1141, https://doi.org/10.1175/JAS-D-14-0370.1.

Torri, G., and Z. Kuang, 2019: On cold pool collisions in tropical boundary layers. Geophys. Res. Lett., 46, 399-407, https://doi. org/10.1029/2018GL080501.

Vallis, G. K., 2017: Atmospheric and Oceanic Fluid Dynamics. Cambridge University Press, 946 pp.

_ - G. Shutts, and M. Gray, 1997: Balanced mesoscale motion and stratified turbulence forced by convection. Quart. J. Roy. Meteor. Soc., 123, 1621-1652, https://doi.org/10.1002/qj.49712354209.

Van Sang, N., R. K. Smith, and M. T. Montgomery, 2008: Tropical-cyclone intensification and predictability in three dimensions. Quart. J. Roy. Meteor. Soc., 134, 563-582, https://doi. org/10.1002/qj.235.

Wang, Y., 2002a: Vortex Rossby waves in a numerically simulated tropical cyclone. Part I: Overall structure, potential vorticity, and kinetic energy budgets. J. Atmos. Sci., 59, 1213-1238, https://doi. org/10.1175/1520-0469(2002)059<1213:VRWIAN>2.0.CO;2.

_ 2002b: Vortex Rossby waves in a numerically simulated tropical cyclone. Part II: The role in tropical cyclone structure and intensity changes. J. Atmos. Sci., 59, 1239-1262, https://doi.org/10.1175/ 1520-0469(2002)059<1239:VRWIAN > 2.0.CO;2.

— , and G. J. Holland, 1995: On the interaction of tropical-cyclonescale vortices. IV: Baroclinic vortices. Quart. J. Roy. Meteor. Soc., 121, 95-126, https://doi.org/10.1002/qj.49712152106.

—, L. Zhang, J. Peng, and S. Liu, 2018: Mesoscale horizontal kinetic energy spectra of a tropical cyclone. J. Atmos. Sci., $\mathbf{7 5}$, 3579-3596, https://doi.org/10.1175/JAS-D-17-0391.1.

— C. A. Davis, and Y. Huang, 2019: Dynamics of lower-tropospheric vorticity in idealized simulations of tropical cyclone formation. J. Atmos. Sci., 76, 707-727, https://doi.org/10.1175/ JAS-D-18-0219.1.

Wing, A. A., S. J. Camargo, and A. H. Sobel, 2016: Role of radiative-convective feedbacks in spontaneous tropical cyclogenesis in idealized numerical simulations. J. Atmos. Sci., 73, 2633-2642, https://doi.org/10.1175/JAS-D-15-0380.1.

Winters, K. B., P. N. Lombard, J. J. Riley, and E. A. D'Asaro, 1995: Available potential energy and mixing in density-stratified fluids. J. Fluid Mech., 289, 115-128, https://doi.org/10. 1017/S002211209500125X.

Wissmeier, U., and R. K. Smith, 2011: Tropical cyclone convection: The effects of ambient vertical vorticity. Quart. J. Roy. Meteor. Soc., 137, 845-857, https://doi.org/10.1002/qj.819.

Würsch, M., and G. C. Craig, 2014: A simple dynamical model of cumulus convection for data assimilation research. Meteor. Z., 23, 483-490, https://doi.org/10.1127/0941-2948/ 2014/0492.

Yang, B., and Z.-M. Tan, 2020: Interactive radiation accelerates the intensification of the midlevel vortex for tropical cyclogenesis. J. Atmos. Sci., 77, 4051-4065, https://doi.org/10.1175/ JAS-D-20-0094.1. 